\title{
Chiral rings in $\mathbf{N}=2$ superconformal theories
}

\section{Citation}

Lerche, Wolfgang, Cumrun Vafa, and Nicholas P. Warner. 1989. "Chiral Rings in $\mathrm{N}=2$ Superconformal Theories." Nuclear Physics B 324 (2): 427-74. https:// doi.org/10.1016/0550-3213(89)90474-4.

\section{Permanent link}

http://nrs.harvard.edu/urn-3:HUL.InstRepos:41385047

\section{Terms of Use}

This article was downloaded from Harvard University's DASH repository, and is made available under the terms and conditions applicable to Other Posted Material, as set forth at http:// nrs.harvard.edu/urn-3:HUL.InstRepos:dash.current.terms-of-use\#LAA

\section{Share Your Story}

The Harvard community has made this article openly available.

Please share how this access benefits you. Submit a story.

\section{Accessibility}




\title{
CHIRAL RINGS IN $\boldsymbol{N}=2$ SUPERCONFORMAL THEORIES
}

\author{
Wolfgang LERCHE
}

California Institute of Technology, Pasadena, CA 91125, USA

Cumrun VAFA

Lyman Laboratory of Physics, Harvard University, Cambridge, MA 02138, USA

Nicholas P. WARNER

Mathematics Department, Massachusetts Institute of Technologv, Cambridge, MA 02139, USA

Received 15 March 1989

\begin{abstract}
We investigate the properties of chiral operators in $N=2$ superconformal theories. In particular, we study the spectral flow of such models under a one-parameter family of twists generated by the $U(1)$ current, and use this to deduce various properties of the ring of chiral primary fields. We furthermore investigate under what conditions a given superconformal theory can be represented as the fixed point of an $N=2$ Landau-Ginzburg theory and show how to determine the superpotential. We also investigate the coset models of Kazama and Suzuki and find a simple cohomological characterization for the elements of the chiral primary ring. Moreover we show how some of them can be represented as LG models.
\end{abstract}

\section{Introduction}

The conformal models in two dimensions possessing $N=2$ world-sheet supersymmetry form a special class of conformal theories, that is, they comprise the only known solutions to string theory at the perturbative level. It has also recently become clear from the connection between $N=2$ theories, renormalization group flows and singularity (catastrophe) theory [1-3] that the $N=2$ theories are, in a sense, the simplest types of conformal theories.

We will first review and extend results of refs. [4-6] exhibiting some general properties that follow from the presence of the $N=2$ superconformal symmetry. In sect. 3, we establish a deep connection between cohomology rings and the ring of chiral primary operators. We then use these results to discuss certain aspects of $N=2$ Landau-Ginzburg models that are based on singularity theory. In particular, we find the conditions for an $N=2$ superconformal model to be representable as the fixed point of a Landau-Ginzburg theory. We next discuss some aspects of the 
coset models constructed by Kazama and Suzuki [7]. We relate the chiral primary states in these models to affine Lie algebra cohomology classes represented by harmonic forms and show that (at least) a subclass of the Kazama-Suzuki models can be represented by Landau-Ginzburg models. We show that in some cases the corresponding superpotentials have a relation to the cohomological properties of the coset manifold. In a subsequent paper we will elaborate on this connection [8]. In the appendix we prove a non-cancellation theorem needed in the study of chiral rings in coset models.

\section{Spectral flow in $N=\mathbf{2}$ superconformal theories}

The $N=2$ superconformal algebra has a very rich structure [4-6]. Of particular importance is the anticommutation relation between the two supersymmetry generators,

$$
\left\{G_{r}^{-}, G_{s}^{+}\right\}=2 L_{r+s}-(r-s) J_{r+s}+(c / 3)\left(r^{2}-1 / 4\right) \delta_{r+s, 0} .
$$

Here, $J$ denotes the $\mathrm{U}(1)$ current of the $N=2$ algebra. This formula is valid for both NS and R sectors, the difference between the two being only that in the NS sector $r, s$ run over half-integral values and in the $\mathrm{R}$ sector over integral values. The other non-trivial commutation relations of the $N=2$ algebra are

$$
\begin{aligned}
& {\left[L_{m}, L_{n}\right]=(m-n) L_{m+n}+(c / 12) m\left(m^{2}-1\right) \delta_{m+n, 0}, } \\
& {\left[L_{n}, G_{r}^{ \pm}\right]=(n / 2-r) G_{n+r}^{ \pm}, } {\left[L_{n}, J_{m}\right]=-m J_{m+n}, } \\
& {\left[J_{m}, J_{n}\right]=(c / 3) m \delta_{m+n, 0}, } {\left[J_{n}, G_{r}^{ \pm}\right]= \pm G_{n+r}^{ \pm} . }
\end{aligned}
$$

The unitarity constraints for the representations of the $N=2$ algebra have been studied in great detail in ref. [4], and we will review some of this work below.

In this paper, all the models will be assumed to have a type $(2,2)$ sypersymmetry, that is, there is an analog of (2.1) and (2.2) for both left- and right-movers. Some of our remarks will however apply equally well to type $(2,0)$ models. We will in addition assume that the left- and right-moving $\mathrm{U}(1)$ charges of each state $\left(q_{\mathrm{L}}, q_{\mathrm{R}}\right)$ satisfy

$$
q_{\mathrm{L}}-q_{\mathrm{R}} \in Z
$$

This condition is not satisfied for all models with $(2,2)$ supersymmetry. A simple set of examples where this is not satisfied is provided by the non-kählerian models of ref. [7] (corresponding to coset models $\mathrm{G} / \mathrm{H}$ with $\operatorname{rank} \mathrm{G}>\operatorname{rank} \mathrm{H}$, for example $\mathrm{G}=\mathrm{SU}(3), \mathrm{H}$ trivial).

Left-chiral states are states in the NS Hilbert space satisfying

$$
G_{-1 / 2}^{+}|\phi\rangle=0,
$$


and anti-chiral states are defined similarly by replacing $G^{+}$with $G^{-}$. Similarly, for right-chiral states, we replace $G$ with $\overline{\mathrm{G}}$. (Note that we are using the terms chiral and anti-chiral in the sense of $N=2$ supersymmetry.) Many of the arguments in this paper are identical for left- and right-movers, and we will only concentrate on the left-movers (in particular, we will denote by $h$ only the left-moving conformal dimension in the following).

Primary chiral states satisfy, in addition to (2.4), the condition

$$
G_{n+1 / 2}^{-}|\phi\rangle=G_{n+1 / 2}^{+}|\phi\rangle=0 \text { for } n \geqslant 0 .
$$

Using (2.5) and (2.4) and the $N=2$ algebra we deduce that for such states

$$
\left\{G_{1 / 2}^{-}, G_{-1 / 2}^{+}\right\}|\phi\rangle=\left(2 L_{0}-J_{0}\right)|\phi\rangle=0 .
$$

Therefore we conclude that for a primary chiral state the dimension $h$ is one-half its charge $q$, i.e. $h=q / 2$. Similarly, for an anti-chiral primary state, we deduce that $h=-q / 2$. In an unitary theory, $\left\{G_{1 / 2}^{-}, G_{-1 / 2}^{+}\right\}$(as well as the corresponding anti-chiral version) is a positive operator (because $G_{-1 / 2}^{+}=G_{1 / 2}^{-\dagger}$ ), and taking its expectation value for any state in the Hilbert space gives the inequality $h \geqslant|q| / 2$. This inequality is saturated precisely for the primary chiral and anti-chiral states. One can also go in the opposite direction and show that the states with $h=q / 2$ are both chiral and primary. To see this, suppose $|\phi\rangle$ satisfies $h=q / 2$, which implies that

$$
\left.\left.\left\langle\phi\left|\left\{G_{1 / 2}^{-}, G_{-1 / 2}^{+}\right\}\right| \phi\right\rangle=0=\left|G_{1 / 2}^{-}\right| \phi\right\rangle\left.\right|^{2}+\left|G_{-1 / 2}^{+}\right| \phi\right\rangle\left.\right|^{2} .
$$

By positivity of the inner product in the Hilbert space we thus have

$$
G_{-1 / 2}^{+}|\phi\rangle=G_{1 / 2}^{-}|\phi\rangle=0 .
$$

To show $|\phi\rangle$ is primary we will have to show (2.5) is also satisfied. First we observe that any operator which lowers the $L_{0}$ eigenvalue, but doe not change the U(1) charge, must annihilate $|\phi\rangle$ to be consistent with the bound $h \geqslant|q| / 2$. In particular, if $J_{m}$ denotes the modes of the U(1) current, we must have

$$
J_{n}|\phi\rangle=0 \text { for } n>0 .
$$

Now we use the $N=2$ commutation relation

$$
\left[J_{n}, G_{r}^{ \pm}\right]= \pm G_{n+r}^{ \pm}
$$

which, combined with (2.7) and (2.8) gives (2.5), as was to be shown. 
Now we will show that any NS state $|\phi\rangle$ with dimension and charge $(h, q)$ can be decomposed as ${ }^{\star}$

$$
|\phi\rangle=\left|\phi_{0}\right\rangle+G_{-1 / 2}^{+}\left|\phi_{1}\right\rangle+G_{1 / 2}^{-}\left|\phi_{2}\right\rangle
$$

where $\left|\phi_{0}\right\rangle$ is a primary chiral state, i.e. it satisfies (2.7). To show (2.9) we consider arbitrary states $\left|\psi_{1}\right\rangle$ and $\left|\psi_{2}\right\rangle$, and vary them so as to minimize the norm

$$
\left|\left(|\phi\rangle-G_{-1 / 2}^{+}\left|\psi_{1}\right\rangle-G_{1 / 2}^{-}\left|\psi_{2}\right\rangle\right)\right|^{2}
$$

That the minimum exists is obvious, because we can restrict attention to $\left|\psi_{1}\right\rangle$ and $\left|\psi_{2}\right\rangle$ in the subsector with dimension and charge $(h-1 / 2, q-1)$ and $(h+1 / 2, q+$ 1) respectively, which for a non-degenerate conformal theory is a finite subspace of the Hilbert space. Let $\left|\phi_{1}\right\rangle$ and $\left|\phi_{2}\right\rangle$ be the corresponding $\left|\psi_{1}\right\rangle$ and $\left|\psi_{2}\right\rangle$ which minimize (2.10). Let

$$
\left|\phi_{0}\right\rangle=|\phi\rangle-G_{-1 / 2}^{+}\left|\phi_{1}\right\rangle-G_{1 / 2}^{-}\left|\phi_{2}\right\rangle
$$

Then the statement that (2.10) is minimized by $\left|\phi_{1}\right\rangle$ and $\left|\phi_{2}\right\rangle$ implies that the norm of $\left|\phi_{0}\right\rangle$ does not change to first order, under arbitrary infinitesimal deformations

$$
\left|\phi_{0}\right\rangle \rightarrow\left|\phi_{0}\right\rangle+G_{-1 / 2}^{+}\left|\epsilon_{1}\right\rangle+G_{1 / 2}^{-}\left|\epsilon_{2}\right\rangle
$$

which implies that $\left|\phi_{0}\right\rangle$ satisfies (2.7) and is a chiral primary state, which therefore establishes the decomposition (2.9).

We now show that if $|\phi\rangle$ is chiral (not necessarily primary), then one can take $\left|\phi_{2}\right\rangle=0$. If we act on (2.9) by $G_{-1 / 2}^{+}$, using the fact that $|\phi\rangle$ is chiral we find that

$$
G_{-1 / 2}^{+} G_{1 / 2}^{-}\left|\phi_{2}\right\rangle=0 \text {. }
$$

Taking the inner product of this state with $\left\langle\phi_{2}\right|$, one sees, by the positivity of the norm, that $G_{1 / 2}^{-}\left|\phi_{2}\right\rangle$ is zero and thus does not contribute to the right-hand side of (2.9). Therefore we may take $\left|\phi_{2}\right\rangle$ to be zero, and $|\phi\rangle$ can be written in the form

$$
|\phi\rangle=\left|\phi_{0}\right\rangle+G_{-1 / 2}^{+}\left|\phi_{1}\right\rangle,
$$

where $\left|\phi_{0}\right\rangle$ is primary and chiral. We will use this result when we discuss LandauGinzburg models in sect. 4.

* This is the analog of the Hodge decomposition for differential forms. See also the discussion in sect. 3 . 
There are also other inequalities that follow from the NS operator algebra of $N=2$ models. For instance, the dimension of a primary chiral field satisfies

$$
h \leqslant c / 6 \text {. }
$$

To show this, use the $N=2$ algebra relation (2.1) in the particular case

$$
\left\{G_{3 / 2}^{-}, G_{-3 / 2}^{+}\right\}=2 L_{0}-3 J_{0}+2 c / 3 \text {. }
$$

If we take the expectation value of this positive operator for any chiral primary state, and use the fact that $h=q / 2$ for such states, we obtain (2.12). We will show below that there always exists a unique chiral primary state in the theory which saturates the bound (2.12). This bound has the following fundamental consequence: since the dimension of primary chiral fields is always less than or equal to $c / 6$, it follows that in non-degenerate $N=2$ conformal theories, for which the spectrum of $L_{0}$ is discrete, there is only a finite number of primary chiral operators.

Now we consider the operator algebra of primary chiral fields. In a general conformal theory we have to worry about how we define the composite operators (i.e. how we subtract leading singularities). However, for chiral primary fields $\phi$ and $\chi$, we can choose the naive product, namely

$$
(\phi \chi)(z)=\lim _{z^{\prime} \rightarrow z} \phi\left(z^{\prime}\right) \chi(z) .
$$

This definition is non-singular without any adjustments by factors of $\left(z-z^{\prime}\right)$. This is so because the $U(1)$ charge of the fields is additive, and the conformal dimensions satisfy

$$
h_{\phi \chi} \geqslant \frac{1}{2}\left(q_{\phi}+q_{\chi}\right)=h_{\phi}+h_{\chi} \text {. }
$$

Note that the product of two chiral fields is again chiral ${ }^{\star}$ though it need not be primary. If $\phi \chi$ is itself primary, this inequality becomes an equality and the singularity in (2.13) (proportional to $\left(z-z^{\prime}\right)^{h_{\phi x}-h_{\phi}-h_{\chi}}$ ) is absent. If $\phi \chi$ is not primary, then the definition (2.13) sets $\phi \chi$ to 0 as $z \rightarrow z^{\prime}$ because of the inequality (2.14). Since there is only a finite number of primary chiral fields, (2.13) defines an interesting finite ring $\star \star \mathscr{R}$ of primary chiral operators ${ }^{\star \star}$. This is the same as the usual operator algebra of chiral fields, modulo setting to zero the descendant chiral

* This can be seen by considering the contour integral of $G^{+}(z)$ about a pair of chiral fields, and noting that the definition of chiral state demands that the contour integral of $G^{+}(z)$ encircling a chiral field vanishes.

* The ring is commutative up to \pm signs, due to the fact that the ring is defined before the GSO projection.

$\star \star \star$ This is not to be confused with the commutative ring which appears for rational conformal field theories [9]. In particular the ring we obtain here is nilpotent, which is not the case for RCFT operator algebra. 
fields. We have to be a little more precise: there are in fact four rings that one can obtain in this way, depending on whether the left- and right-moving states are chiral or anti-chiral primary states. These four rings are pairwise conjugate (by charge conjugation). In the following, unless we state otherwise, we consider one of these four rings. For type $(2,0)$ models we obtain only two rings which are conjugate to one another.

We now consider some aspects of the Ramond sector. If we look at the anticommutator $\left\{G_{0}^{-}, G_{0}^{+}\right\}$, we deduce that $h \geqslant c / 24$ for any state in the $\mathrm{R}$ sector (this is true for the $N=1$ algebra as well). Equality is achieved only for states which are annihilated by both $G_{0}^{+}, G_{0}^{-}$. These are precisely the states which contribute to Witten's index $\operatorname{Tr}(-1)^{F}[10]$.

For theories satisfying (2.3) the operator $(-1)^{F}$, where $F=F_{\mathrm{L}}+F_{\mathrm{R}}$ and $F_{\mathrm{L}}, F_{\mathrm{R}}$ are left- and right-moving fermion numbers, can be defined in terms of the $U(1)$ current as

$$
(-1)^{F}=\exp \left[i \pi\left(J_{0}-\bar{J}_{0}\right)\right] .
$$

The condition (2.3) implies that this is \pm 1 acting on each state. Furthermore, $(-1)^{F}$ commutes with all the bosonic operators in the $N=2$ algebra, and anticommutes with $G^{ \pm}$.

As is well-known [5], one can continuously connect the NS sector to the R sector by considering sectors in which the $\mathrm{U}(1)$ current is twisted. This means that for a twist parameter $\theta$ we consider the Hilbert space $\mathscr{H}_{\theta}$ of states which differ from $\mathscr{H}_{0}$ only in that their $\mathrm{U}(1)$ charge is shifted by $(-c / 3) \theta$. The reason for this choice of normalization is that $J(z) J\left(z^{\prime}\right) \sim c / 3 /\left(z-z^{\prime}\right)^{2}$. This mapping is usually called spectral flow. We will denote the corresponding flow operator by $\mathscr{U}_{\theta}$

$$
\mathscr{U}_{\theta}: \mathscr{H}_{0} \rightarrow \mathscr{H}_{\theta} .
$$

For each operator $O$ acting on $\mathscr{H}_{0}$, there is an operator $O_{\theta}$ acting on $\mathscr{H}_{\theta}$ :

$$
O_{\theta}=\mathscr{U}_{\theta} O \mathscr{U}_{\theta}^{-1}
$$

Under the spectral flow the $N=2$ algebra flows to an isomorphic algebra [5]:

$$
\begin{array}{ll}
\mathscr{U}_{\theta} L_{n} \mathscr{U}_{\theta}^{-1}=L_{n}+\theta J_{n}+(c / 6) \theta^{2} \delta_{n, 0}, & \mathscr{U}_{\theta} J_{n} \mathscr{U}_{\theta}^{-1}=J_{n}+(c / 3) \theta \delta_{n, 0}, \\
\mathscr{U}_{\theta} G_{r}^{+} \mathscr{U}_{\theta}^{-1}=G_{r+\theta}^{+}, & \mathscr{U}_{\theta} G_{r}^{-} \mathscr{U}_{\theta}^{-1}=G_{r-\theta}^{-}
\end{array}
$$

and similarly for the right-movers.

The important feature of the spectral flow is that for $\theta \in Z+1 / 2$ it interpolates between the NS and R sectors, and for $\theta \in Z$ it takes the NS to NS and R to R. One way to see this is to note that the spectral flow is modular transform of twisting the 


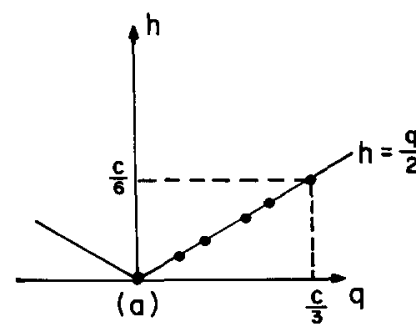

NS - Sector

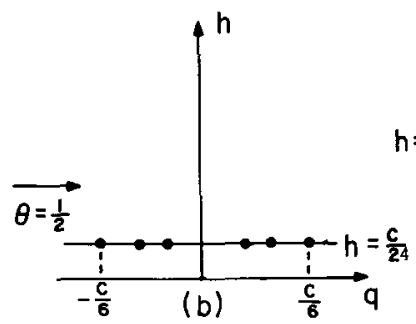

$\mathrm{R}-$ Sector

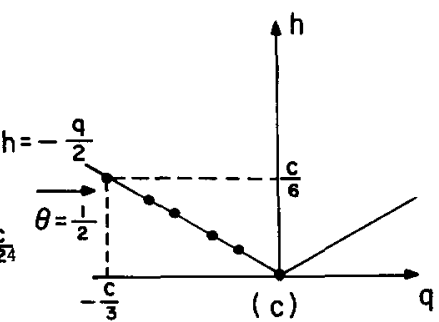

NS - Sector

Fig. 1. In this figure it is shown that starting from the NS sector (a), the chiral primary states flow to the ground states of $\mathrm{R}$ sector (b) with flow parameter $\theta=1 / 2$. Flow by an additional $\theta=1 / 2$ will take the ground state of the Ramond sector to the anti-chiral primary states of the NS sector (c). The effect of the flow on charges of states is simply to shift them.

time direction by $\theta$, which for half-integral values corresponds to twisting boundary conditions by $(-1)^{F}$ (note we have identified left and right spin structures). These modular properties have been considered in [11].

Consider the effect of flow with $\theta=1 / 2$. If we concentrate on the chiral states of the theory, we see that eq. (2.4) becomes

$$
\mathscr{U}_{1 / 2} G_{-1 / 2}^{+} \mathscr{U}_{1 / 2}^{-1} \mathscr{U}_{1 / 2}|\dot{\phi}\rangle=G_{0}^{+}|\tilde{\phi}\rangle=0,
$$

where $|\tilde{\phi}\rangle=\mathscr{U}_{1 / 2}|\phi\rangle$ is in the Ramond sector. If $|\phi\rangle$ is also primary, it follows that (2.5) flows to

$$
G_{n}^{-}|\tilde{\phi}\rangle=G_{n+1}^{+}|\tilde{\phi}\rangle=0 \quad \text { for } \quad n \geqslant 0 .
$$

Combined with (2.17) one sees that $|\tilde{\phi}\rangle$ is annihilated by $G_{0}^{ \pm}$. Therefore, under the flow by $\theta=1 / 2$, the chiral primary states flow to the ground states of the Ramond sector (see fig. 1). Restoring the right-movers we see, from simultaneous spectral flow in the left- and right-moving sectors $\left(\theta_{\mathrm{L}}=\theta_{\mathrm{R}}=\theta\right)$, that there is a one-to-one correspondence between the ground states of the $\mathrm{R}$ sector (for both left and right sectors) and those primary NS states which are simultaneously left- and right-chiral. (This gives an essentially equivalent reason why non-degenerate conformal theories have only a finite number of primary chiral states.)

Furthermore, we note that under this left-right symmetric spectral flow, $q_{\mathrm{L}}-q_{\mathrm{R}}$ does not change. As a consequence, the index

$$
\operatorname{Tr}(-1)^{F}=\underset{\mathbf{R}}{\operatorname{Tr}}\left[(-1)^{J_{0}-\bar{J}_{0}} q^{L_{0}-c / 24} \bar{q}^{\bar{L}_{0}-c / 24}\right],
$$

which receives non-vanishing contributions only from the ground state of the Ramond sector, can be computed in terms of chiral primary states of the NS sector 
by

$$
\operatorname{Tr}(-1)^{F}=\sum_{\mathscr{R}} \exp \left(i \pi\left(q_{\mathrm{L}}-q_{\mathrm{R}}\right)\right)
$$

(The symbol $\mathscr{R}$, which denotes the chiral ring, is not to be confused with $\mathrm{R}$ which denotes the Ramond sector.) This result can be stated in a more general form. Conjugating the operators inside the trace in (2.18) by $\mathscr{U}_{\theta}$ one obtains:

$$
\operatorname{Tr}(-1)^{F}=\operatorname{Tr}_{\mathscr{H}_{\theta}}\left[(-1)^{J_{0}^{\theta}-\bar{J}_{0}^{\theta}} q^{L_{0}^{\theta}-c / 24} \bar{q}^{\theta}-c / 24\right] .
$$

(Note that $J_{0}^{\theta}-\bar{J}_{0}^{\theta}=J_{0}-\bar{J}_{0}$.) In particular, setting $\theta=-1 / 2$, one maps the Ramond sector to the NS sector to yield ${ }^{\star}$

$$
\operatorname{Tr}(-1)^{F}=\operatorname{Tr}_{\mathrm{NS}}\left[(-1)^{J_{0}-\bar{J}_{0}} q^{L_{0}-\frac{1}{2} J_{0}} \bar{q}^{\bar{L}_{0}-\frac{1}{2} \bar{J}_{0}}\right]=\sum_{\mathscr{R}} \exp \left(i \pi\left(q_{\mathrm{L}}-q_{\mathrm{R}}\right)\right) .
$$

In fact one can do better: the only difference between the charges of the chiral primary fields and the charges of the ground state of the Ramond sector is that they are shifted by $c / 6$. Therefore, one can relate the $U(1)$ character valued degeneracy of the Ramond ground states to the character valued sum over the ring $\mathscr{R}$ in the NS sector $\star \star$

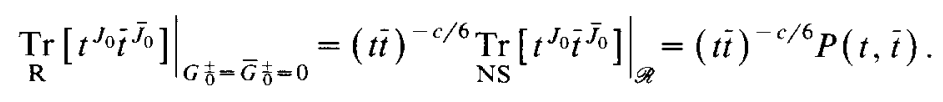

Here, $t$ and $\bar{t}$ can be regarded as independent variables. The relation (2.20) follows as a special case of (2.21) by setting $t=\bar{t}^{-1}=\exp (i \pi)$. Due to charge conjugation invariance of the ground state of the Ramond sector, the relation (2.21) implies that $P(t, \bar{t})=\operatorname{Tr}_{\mathscr{R}} t^{J_{0}} \bar{t}^{\bar{J}_{0}}$ satisfies a certain duality property:

$$
P(t, \bar{t})=(t \bar{t})^{c / 3} P(1 / t, 1 / \bar{t}) .
$$

For reasons that will become clear below we will call $P(t, \bar{t})$ the Poincaré polynomial of the conformal theory. The property (2.22) implies in particular that there exists an unique primary chiral field with the highest possible left and right charges $q_{\mathrm{L}}=q_{\mathrm{R}}=c / 3$ and dimensions $h_{\mathrm{L}}=h_{\mathrm{R}}=c / 6$. This state is the "Poincaré dual" of the unique primary chiral state with $h_{\mathrm{L}}=h_{\mathrm{R}}=0$ (i.e. the vacuum). Under spectral flow to the Ramond sector, these two states become charge conjugates of each other (see fig. 1).

\footnotetext{
${ }^{\star}$ For the behavior of $\operatorname{Tr}(-1)^{F}$ under asymmetric spectral flow see ref. [12].

$\star \star$ These formulas generalize to relations between the full $N=2$ characters for all levels $[11,13]$.
} 
It is clear that if we reversed the direction of the flow, we will get an isomorphism between the (anti-chiral, anti-chiral) primary states and the ground states of the Ramond sector.

Consider now the flow from the NS sector to the NS sector with flow parameter $\theta=1$. Under this flow, (chiral, chiral) primary states map to the (anti-chiral, anti-chiral) primary states. In particular, the vacuum, which is primary and is chiral as well as anti-chiral (i.e. annihilated by $G_{-1 / 2}^{ \pm}$), flows to an anti-chiral state $|\overline{\boldsymbol{\rho}}\rangle=\mathscr{U}_{1}|0\rangle$, which by eq. (2.16) satisfies

$$
G_{n+1 / 2}^{+}|\bar{\rho}\rangle=G_{n-3 / 2}^{-}|\bar{\rho}\rangle=0 \text { for } n \geqslant 0 .
$$

This implies, by setting $r=-3 / 2, s=3 / 2$ in eq. (2.1) that

$$
\left(2 L_{0}+3 J_{0}+2 c / 3\right)|\bar{\rho}\rangle=0 .
$$

Now since $|\bar{\rho}\rangle$ is both anti-chiral and primary, we have $h_{\rho}=-q_{\rho} / 2$ and we deduce that

$$
h_{\rho}=c / 6
$$

We see that the vacuum flows precisely to the conjugate of the above-mentioned chiral primary state with the highest charge (dimension) and thus (2.12) is saturated for this unique anti-chiral state. The structure of the flow is depicted in fig. 1.

To make the spectral flow more transparent it is convenient to bosonize the U(1) current, by writing

$$
J(z)=i \sqrt{c / 3} \partial \phi
$$

(and similarly for $\bar{J}$ ). The normalization in eq. (2.25) is fixed by eqs. (2.1) and (2.2) which are, in turn, a consequence of the requirement that $G^{ \pm}$have \pm 1 unit of charge. A state in the theory with charge $\left(q_{\mathrm{L}}, q_{\mathrm{R}}\right)$ can be represented by

$$
O_{q_{\mathrm{L}}, q_{\mathrm{R}}}=\exp \left[i \sqrt{3 / c}\left(q_{\mathrm{L}} \phi_{\mathrm{L}}-q_{\mathrm{R}} \phi_{\mathrm{R}}\right)\right] \chi
$$

where $\chi$ is a neutral operator. The Hilbert space $\mathscr{H}_{\theta}$ is obtained by shifting the $\phi$ momentum of any state of $\mathscr{H}_{0}$ by $\sqrt{c / 3} \theta$. This means that if we have any state with charge $\left(q_{\mathrm{L}}, q_{\mathrm{R}}\right)$, possibly tensored with other degrees of freedom, it flows to a state with charge $\left(q_{\mathrm{L}}-c \theta / 3, q_{\mathrm{R}}-c \theta / 3\right)$, with $\phi$ momenta $\sqrt{3 / c}\left(q_{\mathrm{L}}-c \theta / 3, q_{\mathrm{R}}-\right.$ $c \theta / 3$ ), tensored with the same degrees of freedom. Therefore the spectral flow operator is

$$
\mathscr{U}_{\theta}=\exp \left(-i \theta \sqrt{c / 3}\left(\phi_{\mathrm{L}}-\phi_{\mathrm{R}}\right)\right)
$$


In particular, we identify the state $|\rho\rangle$ as the spectral flow of the vacuum with $\theta=-1$ with the state of (left-right) momentum $\sqrt{c / 3}$ and dimension $c / 6$. It follows that it is a state purely in the U(1) sector and expressible in terms of free bosons:

$$
\rho=\exp \left[i \sqrt{c / 3}\left(\phi_{\mathrm{L}}-\phi_{\mathrm{R}}\right)\right]
$$

It is clear that the field $\rho$ generates integral units of left-right symmetric spectral flow.

So far we have been quite general in considering $N=2$ superconformal models. For string applications, we are interested in obtaining a space-time supersymmetric solution. The prime examples of conformal theories with $N=2$ supersymmetry are $\sigma$-models on Calabi-Yau manifolds. Quite generally, whether or not the conformal theory comes from a $\sigma$-model, it was shown in ref. [6] that the condition for the existence of space-time supersymmetry is the existence of an $N=2$ superconformal algebra with integral left U(1) charges in the NS sector. This in particular implies that the central charge $c$ must be a multiple of 3 [because $|\rho\rangle$ has charge $(c / 3, c / 3)]$. Set $d=c / 3$. The integrality of the $U(1)$ charges allows us to define the $(-1)^{F_{\mathrm{L}}}$ and $(-1)^{F_{\mathrm{R}}}$ separately in terms of the currents as

$$
(-1)^{F_{\mathrm{L}}}=\exp \left[i \pi J_{0}\right], \quad(-1)^{F_{\mathrm{R}}}=\exp \left[-i \pi \bar{J}_{0}\right] .
$$

In such cases we can perform spectral flow for left- and right-movers independently. For example, we can set $\theta_{\mathrm{R}}=0$ and $\theta_{\mathrm{L}}=1 / 2$. This flows the $(\mathrm{R}, \mathrm{R})$ sector to (NS, R) sector. The corresponding operator can be represented after bosonizing the $\mathrm{U}(1)$ current by

$$
\exp \left[(-i / 2) \sqrt{c / 3} \phi_{\mathrm{L}}\right]
$$

To make this a well-defined operator in the entire theory, (2.28) must be augmented by similar flow operators in the ghost and space-time sectors. This complete operator is well-defined as a consequence of charge integrality and imposing the GSO projection to states with a definite sign of $(-1)^{F_{\mathrm{L}}}$. As such an operator can be used to construct the supersymmetry charge, it was found necessary in ref. [6] to impose integrality of the $\mathrm{U}(1)$ charges to obtain space-time supersymmetry.

For $\left(\theta_{\mathrm{L}}, \theta_{\mathrm{R}}\right)=(-1,0)$, the vacuum flows to a (unique) state in the (chiral, chiral) ring with charge $(d, 0)$ (note that $c=3 d$ ). Similarly, by flowing with $(0,-1)$ we see that there is one and only one (chiral, chiral) state with charge $(0, d)$. These two states correspond to the local operators $\rho_{\mathrm{L}}=\exp \left[i \sqrt{c / 3} \phi_{\mathrm{L}}\right]$ and $\rho_{\mathrm{R}}=$ $\exp \left[-i \sqrt{c / 3} \phi_{\mathrm{R}}\right]$ that generate independent, integral spectral flows in the left- and right-moving sectors ${ }^{\star}$. Obviously, $\rho=\rho_{\mathrm{L}} \otimes \rho_{\mathrm{R}}$.

* That these two states must be in the theory (before the GSO projection) should follow from modular invariance and the fact that $\rho_{\mathrm{L}}$ and $\rho_{\mathrm{R}}$ are local with respect to all fields in the theory. 
As we had mentioned before, for a general $N=2$ superconformal model there are four types of rings arising from the various combinations of chiral and anti-chiral, and left and right. We will denote these rings by $(c, c),(a, a),(c, a),(a, c)$. They are pairwise conjugate. In certain $N=2$ models, such as Landau-Ginzburg models, one obtains only the $(c, c)$ and its conjugate $(a, a)$ ring. For such models, the $(a, c)$ and $(c, a)$ rings are trivial and consist only of the identity operator. However, for theories with integral $\mathrm{U}(1)$ charges, there is a non-trivial relationship between $(c, c)$ and $(a, c)$. In particular, with spectral flow by $\left(\theta_{\mathrm{L}}, \theta_{\mathrm{R}}\right)=(1,0)$ starting from the $(c, c)$ elements in the (NS, NS) sector, we flow to $(a, c)$ elements again in the (NS, NS) sector. This implies, in particular, that if $\chi \in(c, c)$ then

$$
\exp \left[-i \sqrt{c / 3} \phi_{\mathrm{L}}\right] \chi \in(a, c) .
$$

We can define a Poincaré polynomial for $(a, c)$ fields as well, by

$$
P_{(a, c)}=\left.\operatorname{Tr}_{\mathrm{NS}}\left[t^{-J_{0}} \bar{t}^{\bar{J}_{0}}\right]\right|_{(a, c)}
$$

(the sign of the exponent of $t$ is chosen so that we have only positive powers of $t$ and $\bar{t}$ ). Because of the relation between $(c, c)$ and $(a, c)$, the Poincare polynomial of $(a, c)$ is determined in terms of that of $(c, c)$. More precisely, if

$$
P_{(c, c)}=\sum_{p, q=0}^{d} b_{p, q} t^{p} \bar{t}^{q}
$$

where $b_{p, q}$ denotes the number of chiral primary fields with charge $(p, q)$, we must have

$$
P_{(a, c)}=\sum_{p, q=0}^{d} b_{d-p, q} t^{p^{\bar{t} q}}
$$

In order to illustrate these somewhat abstract ideas we shall consider two simple examples. The simplest $N=2$ superconformal model with $c=1$ can be realized by a free boson $\phi$ on a circle with specific radius [14]; this boson can be identified with that in eq. (2.25). The circle has a radius such that the allowed winding (momentum) modes are of the form

$$
\exp \left[i\left(n_{\mathrm{L}} \phi_{\mathrm{L}}-n_{\mathrm{R}} \phi_{\mathrm{R}}\right) / \sqrt{12}\right],
$$

with $n_{\mathrm{L}}-n_{\mathrm{R}}=0 \bmod 6$ (before the GSO projection). The Ramond sector corresponds to odd $n_{\mathrm{L}}, n_{\mathrm{R}}$. The $N=2$ algebra is realized by

$$
\begin{aligned}
G^{ \pm} & =\exp \left[ \pm i \sqrt{3} \phi_{\mathrm{L}}\right], & & \bar{G}^{ \pm}=\exp \left[ \pm i \sqrt{3} \phi_{\mathrm{R}}\right], \\
J(z) & =i \sqrt{1 / 3} \partial \phi, & & \bar{J}(\bar{z})=-i \sqrt{1 / 3} \bar{\partial} \phi .
\end{aligned}
$$


It is easy to see that the only primary chiral states are the vacuum and the state with $\left(n_{\mathrm{L}}, n_{\mathrm{R}}\right)=(2,2)$. This state has dimension $h_{\mathrm{L}}=q_{\mathrm{L}} / 2=h_{\mathrm{R}}=q_{\mathrm{R}} / 2=1 / 6$. We denote the field corresponding to this state by $x$. It has to be identified with the highest dimension primary chiral field $\rho$ discussed above, as it saturates the bound $h=c / 6=1 / 6$. The ring structure is very simple:

$$
\mathscr{R}=\{1, x(\text { subject to } x \cdot x=0)\} .
$$

The vanishing equation can be understood by noting that the dimension of the field $x^{2}$ [identifiable with the state $\left(n_{\mathrm{L}}, n_{\mathrm{R}}\right)=(4,4)$ ] is $2 / 3$, which is higher than twice the dimension of $x$; therefore the naive product of $x$ with itself vanishes, as discussed before. The ground state of the Ramond sector with left-right dimension $(1 / 24,1 / 24)$ is doubly degenerate, and corresponds to $\left(n_{\mathrm{L}}, n_{\mathrm{R}}\right)=( \pm 1, \pm 1)$.

The spectral flow is realized by $\left(n_{\mathrm{L}}, n_{\mathrm{R}}\right) \rightarrow\left(n_{\mathrm{L}}-2 \theta, n_{\mathrm{R}}-2 \theta\right)$. For $\theta=1 / 2$, the chiral primary states flow to the ground states of the $\mathrm{R}$ sector:

$$
(0,0) \rightarrow(-1,-1), \quad(2,2) \rightarrow(1,1) .
$$

Under a spectral flow by $\theta=1$, the chiral primary states go to anti-chiral primary states:

$$
(0,0) \rightarrow(-2,-2), \quad(2,2) \rightarrow(0,0) .
$$

Thus, the vacuum flows to the primary anti-chiral state with the highest dimension. Finally we note that $\operatorname{Tr}(-1)^{F}=2$ as follows from (2.19), and the Poincaré polynomial for this theory is easily seen to be

$$
P(t, \bar{t})=1+(t \bar{t})^{1 / 3} .
$$

The duality (2.22) of the Poincaré polynomial is easily checked in this example. The $(a, c)$ ring is trivial and consists only of the identity operator. We shall return to this example when we discuss Landau-Ginzburg models in sect. 4.

Our second example is the simplest type of superstring compactification, namely compactification on a two dimensional torus (i.e. one complex dimension). This theory has central charge $c=3$, coming from one complex boson $x$ (contributing 2) and one complex fermion $\psi$ (contributing 1). The $N=2$ superalgebra is realized by

$$
G^{+}(z)=\psi^{*} \partial x, \quad G^{-}(z)=\psi \partial x^{*}, \quad J(z)=\psi^{*} \psi
$$

and similarly for the right-movers. It is easy to identify the (left) primary chiral states as:

$$
|0\rangle, \quad \psi_{-1 / 2}^{*}|0\rangle,
$$

and similarly for the right chiral states and for anti-chiral primary states. Note that 
the chiral state above has charge +1 and dimension $1 / 2$ as required for a primary chiral state. The (chiral, chiral) primary states are $|0\rangle, \psi_{-1 / 2}^{*}|0\rangle, \bar{\psi}_{-1 / 2}^{*}|0\rangle$, $\psi_{-1 / 2}^{*} \bar{\psi}_{-1 / 2}^{*}|0\rangle$, and the corresponding $(c, c)$ ring is

$$
\mathscr{R}=\left\{1, \psi^{*}, \bar{\psi}^{*}, \psi^{*} \bar{\psi}^{*}\right\} \text {. }
$$

The Poincaré polynomial is

$$
P(t, \bar{t})=1+t+\bar{t}+t \bar{t}=(1+t)(1+\bar{t}) .
$$

The ring structure is obvious in this example. Also the duality (2.22) of the Poincaré polynomial is easily checked. The index $\operatorname{Tr}(-1)^{F}$ can be computed by setting $t=\bar{t}=-1$ which gives $\operatorname{Tr}(-1)^{F}=0$. Note that the dimension of the unique highest chiral field is $(1 / 2,1 / 2)$, as required by our general arguments; the fields with dimensions $(1 / 2,0)$ and $(0,1 / 2)$ generate integral flows for the left and right Hilbert spaces independently. Finally, the $(a, c)$ ring is:

$$
\mathscr{R}=\left\{1, \psi, \bar{\psi}^{*}, \psi \bar{\psi}^{*}\right\} .
$$

In this example the Poincaré polynomial and the ring structure for the $(c, c)$ and $(a, c)$ primary fields are isomorphic. This is however not true in general.

The ground states of the Ramond sector and its connection with the spectral flow are easy to work out for this example, and we leave it as an instructive exercise to the reader.

\section{Chiral rings and cohomology rings}

We now discuss some geometrical aspects of the chiral rings that we have defined. Quite generally we have defined a ring $\mathscr{R}$ of left-right chiral primary operators, and a natural question arises whether or not this ring has any geometrical significance ${ }^{\star}$. The reader may have noticed that the Poincaré polynomial for the torus model (2.31) is in fact identical with the complex Poincaré polynomial for the torus. In general, the Poincaré polynomial for a complex manifold $\mathrm{M}$ is defined by

$$
P(t, \bar{t})=\sum_{p, q=0}^{d} b_{p, q} t^{p} \bar{t}^{q}
$$

where $d=\operatorname{dim}_{\mathrm{c}} \mathrm{M}$ is the complex dimension of $\mathrm{M}$ and

$$
b_{p, q}=\operatorname{dim} \mathrm{H}^{p, q}(\mathrm{M})
$$

\footnotetext{
* We have greatly benefitted from discussions with $\mathrm{B}$. Greene in preparing this section.
} 
and $\mathrm{H}^{p, q}(\mathrm{M})$ are the Dolbeault cohomology groups. Moreover, the ring structure for the superconformal model on the torus is identical with the ring structure of the Dolbeault cohomology groups of the torus: the primary chiral fields are in one-to-one correspondence with $1, \mathrm{~d} z, \mathrm{~d} \bar{z}, \mathrm{~d} z \wedge \mathrm{d} \bar{z}$, so that $\mathscr{R}$ corresponds to the cohomology ring $\left\{\mathbf{H}^{0,0}, \mathbf{H}^{0,1}, \mathbf{H}^{1,0}, \mathrm{H}^{1,1}\right\}$. The duality noted in (2.22) translates precisely to the Poincaré duality of the cohomology groups:

$$
b_{p, q}=b_{d-p, d-q} .
$$

In fact, the statements that $\operatorname{dim} \mathrm{H}^{d, d}=\operatorname{dim} \mathrm{H}^{0,0}=1$ correspond to something that we know in the $N=2$ theories, namely the uniqueness of the vacuum and of the field $\rho$ with highest charge $(c / 3, c / 3)=(d, d)$. Also for Calabi-Yau manifolds, the statement that $\operatorname{dim} \mathrm{H}^{d, 0}=\operatorname{dim} \mathrm{H}^{0, d}=1$ has the analogue in terms of the unique chiral fields $\rho_{\mathrm{L}}$ and $\rho_{\mathrm{R}}$ defined in the previous section: these are precisely the constant (anti-)holomorphic $d$-form fields that exist on any Kähler manifold with vanishing first Chern class.

The relationship between cohomology and chiral rings is not an accident. If we are considering a supersymmetric non-linear $\sigma$-model based on a manifold $M$, we know from the work of Witten [10] that there is a one-to-one correspondence between the cohomology classes of the manifold and the ground states of the Ramond sector. This was shown by relating the Ramond states to the differential forms on $\mathrm{M}$, and the Ramond-Ramond operator to the deRham cohomology of $\mathrm{M}$. Moreover, when $\mathrm{M}$ is a Kähler manifold (and thus the $\sigma$-model has $N=2$ supersymmetry), the $\partial$ operator corresponds to $G_{0}^{+}$and $\bar{\partial}$ corresponds to $\bar{G}_{0}^{+}$. Since the left-right U(1) currents are of the form $\psi^{*} \psi$ and $\bar{\psi}^{*} \bar{\psi}$, respectively, and $\psi^{*}$ and $\bar{\psi}^{*}$ correspond to wedging with holomorphic and anti-holomorphic differential forms, it follows that the left-right $U(1)$ charges count the holomorphic and anti-holomorphic degrees of the differential forms respectively. So we see that in $N=2$ superconformal models, where the Ramond ground states are related to the chiral primary fields by spectral flow, there is a one-to-one correspondence between the harmonic forms that represent the Dolbeault cohomology of $\mathrm{M}$ and the elements of the ring $\mathscr{R}$. This isomorphism preserves the left-right grading. In particular, this means that the Poincare polynomial we defined for the conformal theory (2.21) is equal to the Poincaré polynomial of Dolbeault cohomology. We can get this isomorphism more directly by identifying $G_{-1 / 2}^{+}$and $\bar{G}_{-1 / 2}^{+}$with $\partial$ and $\bar{\partial}$, respectively.

We now have a one-to-one relation between the elements of the chiral algebra and the cohomology classes. Now we recall that the cohomology groups also possess a ring structure which is compatible with the grading of the forms ${ }^{\star}$. Given the fact

\footnotetext{
^ The ring structure for cohomology is defined by wedging representative forms for each class.
} 
that we have two rings, with the same degeneracies, it is natural to suspect a relation between the two rings. Of course, the fact that the degeneracies are the same, i.e. that the two Poincare polynomials are the same, does not imply that the ring structure is the same, i.e. that the operator algebra of the primary chiral fields is isomorphic to the algebra of the cohomology groups. It is however natural to conjecture a relation between these two rings. (This is related to the question of whether the relations between the Yukawa couplings [15] one finds for the manifold $\mathbf{M}$ are unchanged in the conformal theory; see below.)

If we ignore the higher modes of the string (i.e. ignore the oscillator excitations), the ground states of the Ramond sector (or the chiral primary fields of the NS sector) can be identified with the cohomology elements of the manifold, and the vertex operators of chiral primary fields corresponding to harmonic $(p, q)$ forms can be represented (in the -1 picture) by [16]

$$
b_{i_{1}, \ldots, i_{p}, j_{1}, \ldots j_{q}}\left(x^{i}, x^{* i}\right) \psi^{* i_{1}} \wedge \cdots \wedge \psi^{* i_{p}} \wedge \bar{\psi}^{* j_{1}} \wedge \cdots \wedge \bar{\psi}^{* j_{q}},
$$

where the action of $\psi^{* i}, \bar{\psi}^{* j}$ are represented on the Hilbert space of differential forms by wedging with $\mathrm{d} x^{i}, \mathrm{~d} \bar{x}^{j}$ respectively, and $b$ corresponds to the harmonic $(p, q)$ form. So for large radii, where the semi-classical treatment is reliable, the two rings become isomorphic.

This isomorphism is also manifested in the computation of Yukawa couplings. As was shown in ref. [15], these couplings, at large radii, can be computed from the cohomology algebra of $M$. On the other hand, when $M$ is, for example, a three dimensional Calabi-Yau manifold, one can easily compute the Yukawa couplings from the structure of the rings of chiral fields $[3,17,44]$. For example, the generations corresponding to $(1,1)$ forms correspond to the elements of the ring $(c, c)$ with charges $(1,1)$. This means that they have the correct dimension $\left(\frac{1}{2}, \frac{1}{2}\right)$ in the -1 picture. If $\rho$ has charges $(3,3)$ (and thus is the unique element with that charge), the Yukawa couplings for three fields of charges $(1,1) x, y, z$ are proportional (up to normalization) to $C_{x, y, z}$, where $x y z=C_{x, y, z} \rho$. This is similar for the anti-generations, except that we must now take elements with charge $(-1,1)$.

As we decrease the radii, the semi-classical treatment is no longer justifiable. Indeed, it was shown [18] that for some orbifold models (such as the $Z$ orbifold), the Yukawa couplings do not follow the cohomology structure dictated by the manifold, and therefore the ring of primary chiral fields is not isomorphic to the cohomology ring of the manifold (even after smoothing out orbifold singularities). It was shown [19] that quite generally instanton corrections will affect some of the relations the Yukawa couplings satisfy; in the language discussed here, this means that these corrections invalidate the semi-classical isomorphisms of the two rings. So we see that non-linear sigma models on manifolds which give rise to $N=2$ superconformal models can have a ring structure that is a deformation of the ring structure of the manifold. Nevertheless, we believe that there should be some relation between the 
ring defined by the conformal theory, and some intrinsic geometrical properties of M. In fact, attempts to extend the semi-classical argument just mentioned to the general case, in which one cannot ignore the oscillator modes, naturally leads us to consider the cohomology ring of the loop space over $\mathbf{M}$. In this setting one would be identifying primary chiral fields with the cohomology elements of the loop space, and the ring structure (that is, the operator product algebra) would correspond to wedging differential forms on loop space. It is an extremely interesting question to settle the exact relation of the ring structure of a conformal theory, with such a picture.

As discussed in the previous section there are actually two (up to complex conjugation) inequivalent rings of interest: $(c, c),(a, c)$. As was explained above, for superconformal models coming from compactification on Calabi-Yau manifolds, the $(c, c)$ ring becomes isomorphic to the structure of the cohomology ring of the manifold in the large radius limit. Of course it should be clear in general that it is a matter of convention as to which ring we call $(a, c)$ and which ring we call $(c, c)$ because we can always flip the relative sign of the $J$ and $\bar{J}$, and change our conventions ${ }^{\star}$. So more precisely we should say that one of the two rings $(c, c)$ or $(a, c)$ is a deformation of the cohomology ring of the manifold. One of them gives the Poincaré polynomial of the manifold (2.29) and the other gives a Poincaré polynomial of the form (2.30), which in general differs from the Poincaré polynomial of the manifold. One would clearly like to have a geometric interpretation of the other Poincaré polynomial. One possibility might be that this polynomial is the Poincaré series for (a deformation of) the cohomology ring of another manifold. This is quite possible in light of the fact that string propagation on topologically distinct manifolds can be isomorphic. This happens, for example, for certain orbifolds. If so, there must be another manifold $\tilde{M}$ for which the betti numbers satisfy

$$
b_{p, q}^{\tilde{\mathrm{M}}}=b_{d-p, q}^{\mathrm{M}} .
$$

One other interesting question that arises is that from the work of Dixon [20] we know that we can use charge $(1,1)$ elements of $(c, c)$ and $(-1,1)$ elements of $(a, c)$ to deform the conformal theory; these correspond to massless moduli of the conformal theory. It would be of interest to know whether such deformations would change the ring structures that we have been discussing. Even though they obviously do not change the degeneracies of the ring elements, they do in general change the ring structure. However, it was shown in ref. [21] that if we use the moduli corresponding to charge $(1,1)$ elements of $(c, c)$, the Yukawa couplings of the $(a, c)$ anti-generations do not change. This implies that at least some part of the ring structure of $(a, c)$ is unchanged. In fact, one can easily generalize this result and

^ This remark and the following point have been raised in ref. [20]. 
show that the whole ring structure of $(a, c)$ is unchanged. This in particular means that the semi-classical analysis at large radii should suffice to determine the full ring structure of $(a, c)$. It would be interesting to investigate the geometrical meaning of this. Similarly one can show that the deformations of the moduli corresponding to the charge $(-1,1)$ elements of $(a, c)$ do not change the $(c, c)$ ring structure.

\section{4. $N=2$ Landau-Ginzburg models and catastrophe theory}

One of the aims of the work [1] (see also [3]) was to initiate a classification of $N=2$ superconformal theories by proceeding as follows ${ }^{\star}$ : consider a number of chiral superfields $\Phi_{i}, i=1, \ldots, n$, with $D^{+} \Phi_{i}=\bar{D}^{+} \Phi_{i}=0$, and take any superpotential $W\left(\Phi_{i}\right)$ which has an isolated quasi-homogeneous singularity at $\Phi_{i}=0$. That is, take a holomorphic function for which

$$
W\left(\lambda^{w_{i}} \Phi_{i}\right)=\lambda^{d} W\left(\Phi_{i}\right),
$$

where we take $w_{i}, d$ to be integers with no common factors. The notion of an isolated singularity means that if we set $\partial_{i} W\left(\Phi_{j}\right)=0$ for all $i$, the only solution is at the origin. In other words, we assume that $W$ has no flat directions near the origin. (If we allowed for a line of critcality near the origin, we would obtain a degenerate conformal theory, similar to string theory on non-compact spaces.) The basic hypothesis in ref. [1] is that there exists a choice of $D$-term $K\left(\Phi_{i}, \bar{\Phi}_{i}\right)$ for which the lagrangian

$$
\mathscr{L}=\int \mathrm{d}^{2} z \mathrm{~d}^{4} \theta K\left(\Phi_{i}, \bar{\Phi}_{i}\right)+\left(\int \mathrm{d}^{2} z \mathrm{~d}^{2} \theta W\left(\Phi_{i}\right)+\text { c.c. }\right)
$$

is conformally invariant. The idea is to use $W$ to define a universality class of Landau-Ginzburg theories under the renormalization group flow, the fixed point of which is a conformal theory. Under this flow, the $D$-term adjusts itself so that at the fixed point (4.2) is a conformal theory. The simplification for $N=2$ theories (as compared to $N=0,1$ theories) comes from the assumption that the superpotential does not change during the flow and is thus an invariant of the flow. This assumption has at best only been proven perturbatively. However, one also needs a non-perturbative non-renormalization theorem of $N=2$ theories in two dimensions and such a theorem has not yet been established. Nevertheless the conclusions one

* The renormalization group flows for Landau-Ginzburg models and the importance of non-renormalization theorems in the context of minimal $N=2$ models were first considered in ref. [22]. In addition in that reference there was a conjectured equivalence of the superpotential of the LandauGinzburg models and the defining equation for certain models of [23]. This conjecture was extended and checked for all models of ref. [23] by Greene et al. [2] and Martinec [3] and was established using the ideas of universality of renormalization group flows in ref. [2]. 
reaches using the assumption that for every (isolated) quasi-homogeneous superpotential there exists an $N=2$ conformal theory give further evidence for this hypothesis.

Using the quasi-homogeneity property (4.1), we can read off from the action (4.2) the U(1) charge of the lowest component of $\Phi_{i}$. Since the $\theta$-integrals in the $F$-term have (left, right) charges $(-1,-1)$ and because one requires neutrality of the action, it follows that $W$ has charge $(1,1)$. As a result, $\Phi_{i}$ must have charge $q_{i}=w_{i} / d$ for both its left- and right-moving components. It is easy to see that for any state $q_{\mathrm{L}}-q_{\mathrm{R}}$ is always an integer in Landau-Ginzburg models. This is certainly clear for the field $\Phi_{i}$, as it has equal left-right charges. Moreover, since the most general field is obtained by taking products of $\Phi_{i}$ with $\bar{\Phi}_{i}$, as well as products of their (super)derivatives, it follows that $q_{\mathrm{L}}-q_{\mathrm{R}}$ is always an integer (superderivatives change the charge in integral units). This means that the condition (2.3) is satisfied and our spectral flow arguments apply to Landau-Ginzburg models.

The ring $\mathscr{R}$ of primary chiral operators is isomorphic to the local ring of $W(\Phi)$ : it is simply the space of all monomials of $\Phi_{i}$ modulo setting to zero $\partial_{j} W\left(\Phi_{i}\right)$. (The polynomials $\partial_{j} W\left(\Phi_{i}\right)$ correspond, by the equations of motion, to descendent chiral fields $D^{+} \bar{D}^{+} \bar{\Phi}_{j} \sim \partial_{j} W\left(\Phi_{i}\right)$.) Therefore we have

$$
\mathscr{R}=\frac{C\left[\Phi_{i}\right]}{\left[\partial_{j} W\left(\Phi_{i}\right)\right]} .
$$

The number of elements of the ring is denoted by $\mu=\operatorname{dim} \mathscr{R}$ and is called the multiplicity of $W$.

Since the primary chiral objects have equal left-right charges, it follows that the Poincaré polynomial is only a function of $t \bar{t}$. For convenience we will replace $t \bar{t}$ by the variable $t^{d}$, where $d$ is defined in (4.1). The Poincare polynomial of the superconformal theory becomes then the Poincaré polynomial of the corresponding singularity, and has a particularly simple form [24]:

$$
P(t)=\operatorname{Tr}_{\mathscr{R}}\left[t^{d J_{0}}\right]=\prod_{i=1}^{n} \frac{\left(1-t^{d-w_{i}}\right)}{\left(1-t^{w_{i}}\right)}
$$

One way to see how this formula comes about is to note that if we ignore the numerator of (4.4), it is the partition function of all the chiral fields with no restrictions. Each gradient $\partial_{i} W\left(\Phi_{j}\right)$ is a chiral field with charge $\left(d-w_{i}\right) / d$. Setting to zero the products of a chiral field of this type with all the fields we had originally would subtract from the partition function the partition function of the same states but shifted by the charge $\left(d-w_{i}\right) / d$. The factors of $\left(1-t^{d-w_{i}}\right)$ in the numerator perform this necessary subtraction for each $\partial_{i} W$. The condition that the singularity 
is isolated guarantees that we do not subtract a state twice, i.e. that the gradients of $W$ are independent functions near the origin.

Given any set of numbers $\left(d, w_{i}\right)$, the condition that the right-hand side of (4.4) be a finite polynomial with positive coefficients gives a necessary condition for having a Landau-Ginzburg theory with a quasi-homogeneous potential $W\left(\Phi_{i}\right)$ with $\Phi_{i}$ of charge $w_{i} / d$. This condition is however not sufficient as there are numbers $\left(d, w_{i}\right)$ for which (4.4) is a finite positive polynomial, but there exists no isolated quasi-homogeneous potential with those indices [24].

By l'Hopital's rule, we deduce, using (4.4) and (2.21), that

$$
\operatorname{Tr}(-1)^{F}=P(t=1)=\mu=\prod_{i=1}^{n} \frac{\left(d-w_{i}\right)}{w_{i}}=\prod_{i=1}^{n}\left(\frac{1}{q_{i}}-1\right) .
$$

The chiral primary state $\rho$ with the highest charge can be easily identified using (4.4) by looking at the large $t$ limit of $P(t)$, which gives $t^{\sum_{i}^{d-2 w_{i}}}$. So the charge of $\rho$ is given by $\Sigma\left(d-2 w_{i}\right) / d$, and its conformal dimension is

$$
h_{\rho}=\sum_{i=1}^{n}\left(\frac{1}{2}-q_{i}\right) \equiv \beta .
$$

In the mathematical literature, $\beta$ is known as the singularity index of $W$. From (2.24) it now follows that

$$
c=6 \beta=6 \sum_{i=1}^{n}\left(\frac{1}{2}-q_{i}\right) .
$$

This formula was first proven in ref. [1] by a different method (it was also empirically noticed for the minimal $N=2$ models and tensor products thereof [3]). Because of (4.6), one can always write

$$
P(t)=\sum_{p=0}^{k} b_{p} t^{p}, \quad k=2 d \beta,
$$

where $b_{p}$ counts the number of chiral primary fields of charge $p / d$. Therefore the 'betti' number $b_{d}$ gives the number of continuous moduli of the conformal theory. These deformations are flat [20] and correspond to adding to the superpotential the corresponding chiral primary fields ${ }^{\star}$. Note that given numbers $d, w_{i}$ for which there exists a superpotential of degree $d$ with weights of fields $w_{i}$, one knows the general form of the superpotential. Namely we take any superpotential with these indices,

* It follows that for $c<3$, there cannot be any moduli. 
and add to it $b_{d}$ possible deformations due to massless moduli. Therefore the mere knowledge of the indices $d, w_{i}$ defines the Landau-Ginzburg theory up to a deformation in its moduli.

Note that the number of moduli is different from what is called modality $m$ in the mathematical literature; the latter is given by the number of chiral primary states with charge greater than or equal to one, i.e.

$$
m=\sum_{i \geq d} b_{i}
$$

Poincaré duality takes the usual form:

$$
b_{p}=b_{k-p} .
$$

This follows from (4.4) and is consistent with the general arguments of the first section (2.22). Finally note that in terms of the Landau-Ginzburg fields $\Phi_{i}$, the field with the highest degree $2 \beta$ can be written as

$$
\rho=\operatorname{det}_{i j}\left[\frac{\partial^{2} W(\Phi)}{\partial \Phi_{i} \partial \Phi_{j}}\right] .
$$

The non-vanishing of the determinant is the condition for the singularity to be isolated. It is easy to check that $\rho$ defined above has the right charge.

The question arises as to which subset of all $N=2$ models can be written in terms of Landau-Ginzburg models. As we have discussed, the chiral primary fields in a Landau-Ginzburg theory have all the same sense of chirality for left- and right-movers. Moreover $q_{\mathrm{L}}=q_{\mathrm{R}}$ for all such states. Therefore, theories that have both non-trivial $(c, c)$ and $(a, c)$ primary states cannot be written in the canonical Landau-Ginzburg form. Examples for non-Landau-Ginzburg theories are toroidal compactifications in even dimensions. These theories contain non-trivial primary fields of both chiralities. This is obvious for our $c=3$ example described in sect. 2; not only does it have $(c, c)$ and $(a, c)$ fields, but also the Poincaré polynomial of $(c, c)(2.31)$ is not a function of $(t \bar{t})$ only, as would be the case if $q_{\mathrm{L}}=q_{\mathrm{R}}$ for all primary chiral fields.

More generally, all theories that have integral left and right $\mathrm{U}(1)$ charges (for example, $\sigma$-models on Kähler manifolds with vanishing first Chern class), allow for left-right asymmetric spectral flow giving rise to chiral primary fields which have $q_{\mathrm{L}} \neq q_{\mathrm{R}}$. In particular, the operator $\rho_{\mathrm{L}}$ discussed in sect. 2 is such an example. Thus, these theories cannot be represented as Landau-Ginzburg models. However, orbifolds of Landau-Ginzburg models may well contain both $(c, c)$ and $(a, c)$ states and thus describe such conformal theories; for instance, it is known that conformal theories on Calabi-Yau manifolds can be described as orbifolds of Landau- 
Ginzburg theories [2,3], and these do have both $(c, c)$ and $(a, c)$ states ${ }^{\star}$. If we construct a Calabi-Yau compactification by orbifoldizing a Landau-Ginzburg model, we will get rid of some elements in the ring $(c, c)$ by projecting the untwisted sector onto invariant states. However, in general, we also get new elements in the ring $(c, c)$ coming from the twisted sectors. Prior to twisting there were no non-trivial elements in $(a, c)$, but after orbifoldizing we get some non-trivial elements because, as shown in sect. 3, these correspond to Kähler deformations that are always present ${ }^{\star \star}$. Thus a subring of the $(c, c)$ states of the Landau-Ginzburg theory forms a subring of $(c, c)$ states of the Calabi-Yau theory, and this allows us to compute Yukawa couplings of some of the anti-generations by working entirely in the unorbifoldized Landau-Ginzburg theory. At any rate, all the relations between Yukawa couplings for three fields can be determined from the full ring structure of the orbifoldized Landau-Ginzburg theories, as discussed in sect. 3.

We have seen that an $N=2$ theory arising from a Landau-Ginzburg fixed point necessarily satisfies:

(i) The theory contains only one conjugate pair $((c, c)$ and $(a, a))$ of finite dimensional chiral rings, and these rings contain only left-right symmetric fields with $q_{\mathrm{L}}=q_{\mathrm{R}}$.

It is therefore tempting to conjecture that this is also a sufficient condition for an $N=2$ theory to correspond to a Landau-Ginzburg theory (4.2). This turns out not to be true, as some models of ref. [7] satisfy this condition but are not LG (see sect. 5). However, Landau-Ginzburg theories have some additional important features:

(ii) All (left-right symmetric) chiral fields are generated by a finite set of chiral primary fields $\Phi_{i}$ (and similarly for the anti-chiral fields).

(Note that chiral superderivatives of anti-chiral primary fields can be expressed, via the equations of motion, in terms of chiral fields.)

(iii) All (left-right symmetric) fields in the theory can be obtained by taking arbitrary operator products of $\Phi_{i}$ and $\bar{\Phi}_{i}$.

We now show, by a plausible but not rigorous argument, that any $N=2$ theory, for which (i), (ii) and (iii) are true, has a ring structure $\mathscr{R}$ isomorphic to that of a Landau-Ginzburg model. In other words, we show that $\mathscr{R}$ is of the form (4.3). We do this by explicitly finding $W$. That this is enough to show the equivalence with a Landau-Ginzburg theory is not obvious, but should presumably be a consequence of the hypothesis of ref. [1] which implies that the ring structure of primary chiral fields determines the theory completely. Otherwise we would have a collection of different fixed points for a given superpotential, as has been found in theories with fewer supersymmetries [27].

* Toroidal compactification [25] and orbifolds based on them [26] could also be viewed as orbifolds of Landau-Ginzburg models.

$\star \star$ Our conventions in this section are opposite to that of sect. 3 , where the $(c, c)$ corresponded to Kähler deformations. 
Consider an $N=2$ model satisfying the conditions stated above. We concentrate on the left moving piece of the $(c, c)$ primary fields. Let $\Phi_{i}$ denote the holomorphic parts of the independent generators of the primary chiral fields, and assume that it is normalized so that

$$
\Phi_{i}(z) \bar{\Phi}_{i}\left(z^{\prime}\right) \sim\left(z-z^{\prime}\right)^{-2 h_{i}}+\cdots
$$

We give a naive argument that illustrates the main idea, and subsequently we fill in some (but not all) of the technical gaps. Consider $G^{+}(z)$. This is a chiral field, as follows by noting that $G^{+}(z) G^{+}\left(z^{\prime}\right)$ has no singular piece. The state corresponding to $G^{+}$is

$$
G^{+}(0)|0\rangle=G_{-3 / 2}^{+}|0\rangle \text {. }
$$

(This is, in fact, the left-moving part of one of the two dimensional gravitinos.) Note, however, that $G^{+}$is not a primary chiral field, because it does not satisfy (2.6). Rather, $G^{+}$has $h=3 / 2, q=1$. The assumption that all the chiral fields must be representable as functions of $\Phi_{i}$ implies in particular that $G^{+}=W\left(\Phi_{i}\right)$ for some function $W$. Now, by assumption the ring structure $\mathscr{R}$ is of the form

$$
\mathscr{R}=C[\Phi] / \mathrm{J},
$$

where $\mathbf{J}$ denotes the ideal generated by all polynomials of superfields that vanish as a consequence of (2.13). Take such a polynomial $f$, to which corresponds a chiral state $|f\rangle$ (the state is chiral because the product of chiral fields is again chiral). We now use (2.11) to write

$$
|f\rangle=G_{-1 / 2}^{+}\left|f_{1}\right\rangle
$$

There can be no chiral primary component, $\left|f_{0}\right\rangle$, in (4.9) since this would mean that $|f\rangle$ would not belong to $\mathrm{J}$. Now we use the assumption that all fields are generated by products of $\Phi_{i}$ and $\bar{\Phi}_{j}$, and since $D^{+} \Phi_{i}=0$, using (4.9) we see that the ideal $\mathrm{J}$ is generated by $D^{+} \bar{\Phi}_{i}$. In other words,

$$
\mathscr{R}=C[\Phi] /\left[D^{+} \bar{\Phi}_{i}\right] .
$$

Since $D^{+} \bar{\Phi}_{i}$ is the leading singularity in $G^{+}(z) \bar{\Phi}_{i}\left(z^{\prime}\right)$, and we know that $G^{+}(z)=$ $W(\Phi)$, using (4.8) we see that

$$
D^{+} \bar{\Phi}_{i}=\partial_{i} W(\Phi)
$$

which proves that $\mathscr{R}$ has the ring structure of a Landau-Ginzburg theory. Note that $W$ constructed here has the correct charge to serve as the superpotential of a Landau-Ginzburg theory. Note also that by the homogeneity property of $W$ in a 
LG theory, it is easy to see that it should belong to the ideal $\mathrm{J}$, as is the case for $G^{+}$ which is a descendant chiral state.

We now fill in some of the technical gaps in the foregoing argument. There are two major weaknesses: one is that we have been somewhat cavalier in our treatment of operator products. We will show how this might be remedied. The other weakness is that we should really be considering the left- and right-moving modes at the same time. We will comment on this at the end.

The assumption that all the chiral fields are generated by $\Phi_{i}$ means that if we consider arbitrary non-local operators of the type

$$
F_{i_{1}, \ldots, i_{n}}\left(z_{1}, \ldots, z_{n}\right)=\Phi_{i_{1}}\left(z_{1}\right) \ldots \Phi_{i_{n}}\left(z_{n}\right)
$$

and define

$$
F_{i_{1}, \ldots, i_{n}}^{\lambda}\left(z_{1}, \ldots, z_{n}\right)=F_{i_{1}, \ldots, i_{n}}\left(\lambda z_{1}, \ldots, \lambda z_{n}\right) \text {, }
$$

then, in the limit as $\lambda \rightarrow 0$, after rescaling $F^{\lambda}$ by appropriate negative powers of $\lambda$ and dropping the singular terms, we obtain all chiral fields. The power of $\lambda$ is always negative, as follows from our discussion following (2.14). Suppose we are trying to construct a chiral operator $O_{h}^{q}$ of charge $q$ and dimension $h$. Let $F^{\lambda}$ denote the appropriate linear combination of the $F_{i_{1}, \ldots, i_{n}}\left(\lambda z_{1}, \ldots, \lambda z_{n}\right)$, such that the finite part of $F^{\lambda}$ in the limit $\lambda \rightarrow 0$ yields $O_{h}^{q}$ :

$$
O_{h}^{q}(0)=\lim _{\lambda \rightarrow 0} \lambda^{-(h-q / 2)} F^{\lambda} .
$$

The power of $\lambda$ is determined by matching the conformal dimensions on both sides: since the component fields on the right-hand side are all chiral and primary, their total dimension is $q / 2$, whereas the dimension of the field on the left-hand side is $h$. We have to be a little more precise with the definition (4.11); sometimes there are more singular terms on the right-hand side of (4.11). So what we mean by "lim" is the $\lambda$ independent piece of the right-hand side. The serious shortcoming in the definition (4.11) is that the normalization of $O_{h}^{q}(0)$ depends exactly on where we pick the points $z_{1}, \ldots, z_{n}$. Presumably some kind of averaging is needed to get something independent of the precise locations.

From here on, whenever we write an operator $F\left(\Phi_{i}\right)$ as some polynomial in $\Phi_{i}$, we mean that we first point-split all the operators (including operators raised to some powers) and consider the non-local operators of the type (4.10). Ultimately we are, of course, only interested in local operators which we get from $F$ by the procedure indicated in (4.11). With this definition in mind we now proceed to construct the superpotential $W$.

Consider again $G^{+}(z)$. As discussed above, since this is a chiral field we can construct it in terms of $\Phi_{i}$, and call the corresponding polynomial $W\left(\Phi_{i}\right)$. It follows 
from (4.11) (since the dimension of $G^{+}$is $3 / 2$ and its charge 1), that

$$
G^{+}(0)=\lim _{\lambda \rightarrow 0} \lambda^{-1} W^{\lambda}\left(\Phi_{i}\right)
$$

We showed above that all the descendant chiral fields are generated by $D^{+} \overline{\boldsymbol{\Phi}}_{i}$ (which correspond to chiral non-primary states $G_{-1 / 2}^{+}\left|\bar{\Phi}_{i}\right\rangle$ ). This can be represented by

$$
D^{+} \bar{\Phi}_{i}(0)=\oint G^{+}(z) \bar{\Phi}_{i}(0)
$$

or equivalently, by

$$
D^{+} \bar{\Phi}_{i}(0)=\lim _{\lambda \rightarrow 0} \lambda G^{+}(\lambda) \bar{\Phi}_{i}(0) .
$$

Since $D^{+} \bar{\Phi}_{i}$ is a chiral field (because $\left(D^{+}\right)^{2}=0$ ), it must, by assumption, be representable as a function of $\Phi_{i}$. Let us call that function $H_{i}$. Noting that the dimension and charge of $D^{+} \bar{\Phi}_{i}$ are $h=h_{i}+1 / 2$ and $q=-q_{i}+1=-2 h_{i}+1$, we must have (using (4.11))

$$
D^{+} \bar{\Phi}_{i}=\lim _{\lambda \rightarrow 0} \lambda^{-2 h_{i}} H_{i}^{\lambda}
$$

Now using (4.13), (4.12) and (4.14) it follows that $H_{i}$ must be in the operator product of $\bar{\Phi}_{i}$ and $W(\Phi)$, and it must appear as the term proportional to $\lambda^{-2 h_{i}}$. Using the form (4.12) of $G^{+}$and the normalization (4.8), we see that when we bring $\bar{\Phi}_{i}$ near ${ }^{\star} G^{+}$, the correct singularity structure arises when we take the leading contraction of $\bar{\Phi}_{i}$ with $\Phi_{i}$, and this leaves us with $\partial_{i} W(\Phi)$ in the operator product. This implies that

$$
H_{i}=\partial_{i} W(\Phi)
$$

which completes the argument.

The other weakness in the argument comes from dropping the right-moving piece. This could be remedied by trying to construct $G^{+} \bar{G}^{+}$from the $(c, c)$ fields, rather than dropping the right-moving pieces and repeat the above argument for that case. This however is in general not a left-right symmetric field, and in general cannot be made from the chiral primary fields. For example if we have tensor products of two LG models with chiral supersymmetry generators $G_{1}^{+}, G_{2}^{+}$, then we can construct

$$
G_{1}^{+} \bar{G}_{1}^{+}+G_{2}^{+} \bar{G}_{2}^{+}
$$

* We must assume that the fields which are used to form $G^{+}$are much closer to each other than to $\bar{\Phi}_{i}$. This, however, does not affect our argument. 
using chiral primary fields, but not $G^{+} \bar{G}^{+}$which is

$$
G_{1}^{+} \bar{G}_{1}^{+}+G_{2}^{+} \bar{G}_{2}^{+}+G_{1}^{+} \bar{G}_{2}^{+}+G_{2}^{+} \bar{G}_{1}^{+}
$$

But the argument we gave could be modified to defining $W$ by

$$
\left[G^{+} \bar{G}^{+}\right]_{\mathrm{L}-\mathrm{R} \text { symmetric }}=W\left(\Phi_{i}\right) .
$$

This fills in some of the technical gaps of our argument. It would be nice if the foregoing could be made completely rigorous.

To illustrate the identity $G^{+} \bar{G}^{+}=W$ we return to the example considered in sect. 2 with $c=1$. Recall that in this model the (holomorphic) chiral fields are generated by

$$
x=\exp \left[2 i\left(\phi_{\mathrm{L}}-\phi_{\mathrm{R}}\right) / \sqrt{12}\right]
$$

and in particular,

$$
G^{+} \overline{G^{+}}=\exp \left[6 i\left(\phi_{\mathrm{L}}-\phi_{\mathrm{R}}\right) / \sqrt{12}\right]
$$

Note that $G^{+} \bar{G}^{+}=x^{3}$, which implies that $W=x^{3}$, in accordance with the LandauGinzburg superpotential found in $[1,3]$.

\section{Kählerian coset models}

In this section we will discuss some of the results we have found in the study of chiral rings in the coset models of Kazama and Suzuki [7] and their relation with Landau-Ginzburg models. We will first briefly review these models, and then identify the chiral primary fields. Subsequently we discuss a subclass of these models that can be represented as Landau-Ginzburg theories. We also discuss the connection of the chiral primary ring in this class of models with the cohomology ring of coset manifolds. This study of coset models will be continued (and elaborated) in a subsequent paper [8].

Kazama and Suzuki have studied under what conditions an $N=1$ superconformal coset model $\mathrm{G} / \mathrm{H}$ can have an extra sypersymmetry, to give rise to an $N=2$ superconformal model. Their conclusion is very simple: if rank $G=\operatorname{rank} H$, then the condition needed for $N=2$ supersymmetry is that $\mathrm{G} / \mathrm{H}$ be a Kähler manifold. This in turn is equivalent to the statement that $\mathrm{H}$ is the centralizer (the little group) of a toroidal subgroup of $\mathrm{G}$. For example, if $\mathrm{G}=\mathrm{SU}(m+n)$ and we take the toroidal subgroup to be a $\mathrm{U}(1)$ with $m$ entries equal to a given phase, and $n$ equal to some other phase, we obtain $\mathrm{H}=\mathrm{SU}(m) \times \mathrm{SU}(n) \times \mathrm{U}(1)$. If $\operatorname{rank} \mathrm{G} \neq \operatorname{rank} \mathrm{H}$, these models do not satisfy the condition $q_{\mathrm{L}}-q_{\mathrm{R}} \in Z$, unless they are twisted, and we will not consider such models here. The kählerian $\mathrm{G} / \mathrm{H}$ models of ref. [7], where $\mathrm{G}$ and $H$ have equal rank, contain only states that satisfy $q_{\mathrm{L}}-q_{\mathrm{R}} \in Z$. 
For kählerian spaces, the Lie algebra of $\mathrm{G}$ decomposes as

$$
\boldsymbol{g}=\boldsymbol{h} \oplus \boldsymbol{t}_{+} \oplus \boldsymbol{t}_{-},
$$

with $\boldsymbol{t}_{+}$and $\boldsymbol{t}_{-}$forming closed Lie algebras:

$$
\left[t_{+}, t_{+}\right] \subset t_{+} \quad\left[t_{-}, t_{-}\right] \subset t_{-} .
$$

Among the kählerian models, there is a special class which consists of hermitian symmetric spaces (HSS). This class has the property that the G/H theory has no extra U(1)"s other than the one implied by the presence of $N=2$ superconformal symmetry [7]. For this class, the Lie algebras of $\boldsymbol{t}_{+}$(and $\boldsymbol{t}_{-}$) are abelian.

The $\mathrm{G} / \mathrm{H}$ superconformal model can be decomposed as a level $k$ affine algebra of $\mathrm{G}$, tensored with a level one affine algebra of $\operatorname{SO}(\operatorname{dim}(\mathrm{G} / \mathrm{H}))$, divided by a level $k+g_{\mathrm{G}}-g_{\mathrm{H}}$ representation of affine $\mathrm{H}$, where $g_{\mathrm{G}}$ and $g_{\mathrm{H}}$ denote the dual Coxeter numbers of $G$ and $H$. For convenience we define $d=\frac{1}{2} \operatorname{dim}(G / H)$. The affine algebra of $\mathrm{SO}(2 d)$ at level one will be presented by $d$ complex fermions $\psi^{\alpha}$, where $\alpha$ runs over all the roots of $\boldsymbol{t}_{+}$(with $\psi^{-\alpha}=\psi^{* \alpha}$ ); these fermions could be thought of as providing a basis for the complex tangent vectors to $\mathrm{G} / \mathrm{H}$.

If we denote the currents of $t_{+}$by $J^{\alpha}(z)$, then the $N=2$ chiral supersymmetry generator is given by

$$
\begin{aligned}
G^{+}(z)= & (2 /(k+g))^{1 / 2}\left[\sum_{\alpha \in \boldsymbol{t}_{+}} \psi^{\alpha}(z) J^{-\alpha}(z)\right. \\
& \left.\left.+\frac{1}{2} \sum_{\alpha, \beta, \gamma \in t_{+}} f_{\alpha \beta}^{\gamma} \psi^{\alpha}(z) \psi^{\beta}(z) \psi^{-\gamma}(z)\right)\right],
\end{aligned}
$$

where $f_{\alpha \beta}^{\gamma}$ denote the (suitably normalized) structure constants of $\boldsymbol{t}_{+} ; G^{-}$is defined to be the complex conjugate of the foregoing expression. Here and in the following $g$ will denote the dual coxeter number of $\mathrm{G}$. If we let $H^{i}(z)$ denote the Cartan currents of G, the $N=2 \mathrm{U}(1)$ current is given by

$$
\begin{aligned}
J(z)= & \sum_{\alpha \in \boldsymbol{t}_{+}} \psi^{\alpha}(z) \psi^{* \alpha}(z)-(1 /(k+g)) \\
& \times \sum_{\alpha \in \boldsymbol{t}_{+}}\left[\boldsymbol{\alpha} \cdot\left(H(z)+\sum_{\beta \in \boldsymbol{t}_{+}} \beta \psi^{\beta}(z) \psi^{* \beta}(z)\right)\right] .
\end{aligned}
$$

We wish to investigate the chiral rings in these models. In particular, this will allow us to identify some of them with Landau-Ginzburg theories. From the structure of the coset models of ref. [7], one can see that all the $(c, c)$ states satisfy $q_{\mathrm{L}}=q_{\mathrm{R}}$, and therefore these models have a chance of being represented by Landau-Ginzburg theories. As discussed in sect. 2, by spectral flow, there is a correspondence between primary chiral states and the ground states of the Ramond 
sector. As it turns out, it is more convenient to find the ground states of the Ramond sector and we use this correspondence to simplify our computations. The ground states of the Ramond sector are defined by

$$
G_{0}^{ \pm}|\phi\rangle=0
$$

where

$$
G_{0}^{ \pm}=(2 /(k+g))^{1 / 2}\left[\sum_{\alpha \in t_{+}} \sum_{n} \psi_{n}^{ \pm \alpha} J_{-n}^{\mp \alpha}+\frac{1}{2} \sum_{\alpha, \beta, \gamma \in t_{+}} \sum_{m, n} f_{ \pm \alpha \pm \beta}^{ \pm \gamma} \psi_{n}^{ \pm \alpha} \psi_{m}^{ \pm \beta} \psi_{-(m+n)}^{\mp \gamma}\right]
$$

As discussed in sect. 2 and 3, we can think of $G_{0}^{+}$as analogous to $\partial$ and $G_{0}^{-}$to its adjoint, and in this setup the ground states of the Ramond sector can be thought of as the non-trivial cohomology elements (represented by "harmonic forms"). In order to find the solutions to (5.2) in the coset models, we first have to act with $G_{0}^{ \pm}$ on the Hilbert space of the superconformal $G$ model, and find inequivalent solutions to (5.2). Solutions are equivalent if they differ by a action of $\mathrm{H}$ currents. Note that the Hilbert space of the $G$ model in the Ramond sector consists of representations of the affine $\mathrm{G}$ algebra at a given level $k$, tensored with the two spinor representations of $\mathrm{SO}(2 d)$ at level one. Finding solutions to (5.2) acting on this Hilbert space is not a trivial problem.

As it turns out, the ground states of the $\mathrm{G} / \mathrm{H}$ model do not necessarily come from the ground states of the affine $G$ theory. However, we will first investigate which states among the ground states of the $\mathrm{G} / \mathrm{H}$ model do come from the ground states of the $G$ theory. The ground state of the superconformal $G$ model consists of a finite dimensional representation of $G$ (which we denote by its highest weight vector $\Lambda$ ), tensored with the two fundamental spinors of $\operatorname{SO}(2 d)$. This state is annihilated by the strictly positive modes of currents and fermions in (5.2). Therefore to solve (5.2) for these ground states we are left with the zero mode piece of $G_{0}^{ \pm}$. This truncation is similar to the truncation which was used in ref. [10] to obtain the ground states of two dimensional models, and is similar to the semi-classical approximation we discussed in sect. 3 . Truncating from two to one dimensions means deleting the $z$ dependence from the operators. In particular we have then

$$
\begin{gathered}
G^{+}=(2 /(k+g))^{1 / 2}\left(\sum_{\alpha \in \boldsymbol{t}_{+}} \psi^{\alpha} J^{-\alpha}+\frac{1}{2} \sum_{\alpha, \beta, \gamma \in \boldsymbol{t}_{+}} f_{\alpha \beta}^{\gamma} \psi^{\alpha} \psi^{\beta} \psi^{-\gamma}\right), \\
J(z)=\sum_{\alpha \in \boldsymbol{t}_{+}} \psi^{\alpha} \psi^{* \alpha}-(1 /(k+g)) \sum_{\alpha \in \boldsymbol{t}_{+}}\left[\alpha \cdot\left(H+\sum_{\beta \in \boldsymbol{t}_{+}} \beta \psi^{\beta} \psi^{* \beta}\right)\right],
\end{gathered}
$$


where we can add the subscript zero to the operators if we wished to emphasize their connection to the two dimensional model. It is convenient to think of the spinor representation as obtained by acting with the $\psi^{\alpha}$ on the ground state spinor (taken to be the state $|(-1 / 2, \ldots,-1 / 2)\rangle$, where the $-1 / 2$ 's denote the $\operatorname{SO}(2 d)$ spinor weights). We can then think of the ground states of the fermionic sector as to correspond to the exterior algebra of $\psi^{\alpha}$, which we denote by

$$
\bigwedge t_{+}
$$

If we let $\mathrm{V}_{\Lambda}$ denote the finite dimensional vector space that forms the representation $\Lambda$ of $\mathrm{G}$, then the finite dimensional Hilbert space corresponding to the ground states is

$$
\mathscr{H}=\bigwedge \boldsymbol{t}_{+} \otimes V_{\Lambda} .
$$

For non-linear sigma models, the ground state Hilbert space is obtained from the space of differential forms. Here we can think of $\psi^{\alpha}$ as a basis for holomorphic differential forms, and thus trying to find solutions to $G^{ \pm}=0$ is a cohomology problem. It turns out what we have just defined has been studied extensively by mathematicians [28], and is called the Lie algebra cohomology of $\boldsymbol{t}_{+}$with coefficients in the representation $\Lambda$ of $G$. The cohomology groups (which are in one-to-one correspondence with the solutions to $G^{ \pm}=0$ ) are denoted by

$$
H^{*}\left(\boldsymbol{t}_{+}, V_{A}\right) \text {. }
$$

Of course, the cohomology elements form $\mathrm{H}$ representations, because both $V_{A}$ and the $t_{+}$form representations of $H$, and to identify the states of the $G / H$ theory, we have to decompose $\mathrm{H}^{*}\left(\boldsymbol{t}_{+}, V_{\Lambda}\right)$ to representations of the group $\mathrm{H}$ and pick one state for each irreducible representation of $\mathrm{H}$ (the symbol $\mathrm{H}^{*}$ denoting cohomology groups is not to be confused with $\mathrm{H}$ denoting the $\mathrm{G}$ subgroup). It turns out that the number of such irreducible representations of $\mathrm{H}$ is independent of which representation $A$ of $\mathrm{G}$ we choose, and is equal in number to the ratio of the dimension of the Weyl group of $\mathrm{G}$ to that of $\mathrm{H}$ :

$$
r=|\mathrm{W}(\mathrm{G})| /|\mathrm{W}(\mathrm{H})| .
$$

One can show that the number of irreducible $\mathrm{H}$ representations in $\mathrm{H}^{*}\left(\boldsymbol{t}_{+}, V_{A}\right)$ is greater than or equal to $r$ by employing a simple index argument, which we will now discuss. On the spin representation of $\operatorname{SO}(2 d)$ the character of $\exp \left(2 \pi i \sum_{\alpha} z_{\alpha} \psi^{\alpha} \psi^{* \alpha}\right)$ is given by

$$
\frac{1}{2}\left\{\left[\prod_{\alpha \in \boldsymbol{t}_{+}}\left(\mathrm{e}^{i \pi z_{\alpha}}+\mathrm{e}^{-i \pi z_{\alpha}}\right)\right]+\boldsymbol{\epsilon}\left[\prod_{\alpha \in \boldsymbol{t}_{+}}\left(\mathrm{e}^{i \pi z_{\alpha}}-\mathrm{e}^{-i \pi z_{\alpha}}\right)\right]\right\},
$$


where $\epsilon=+1$ for the $(s)$ spinor and $\epsilon=-1$ for the $(c)$ spinor representations. Now recall that in the embedding of $\mathrm{H}$ into $\mathrm{SO}(2 d)$ the Cartan currents, $H^{i}$, of $\mathrm{H}$ are given by

$$
H^{i}=\sum_{\beta \in t_{+}} \beta^{i} \psi^{\beta} \psi^{* \beta}
$$

and hence the character of $\exp (2 \pi i \mu \cdot \mathrm{H})$ is given by (5.6) with $z_{\alpha}=\alpha \cdot \mu$.

From the Weyl character formula, the character of $G$ in the representation with highest weight $\Lambda$ is given by

$$
\frac{\sum_{w \in \mathrm{W}_{\mathrm{G}}}(w) \mathrm{e}^{-2 \pi i \mu \cdot\left[w\left(\Lambda+\rho_{\mathrm{G}}\right)-\rho_{\mathrm{G}}\right]}}{\prod_{\alpha \in \Delta^{+}(\mathrm{G})}\left(1-\mathrm{e}^{2 \pi i \mu \cdot \alpha}\right)},
$$

where $\rho_{\mathrm{G}}$ is half of the sum of the positive roots of $\mathrm{G}$ and $\Delta^{+}(\mathrm{G})$ denotes the set of positive roots of $\mathrm{G}$. To compute the index of $G_{0}=G_{0}^{+}+G_{0}^{-}$on the Hilbert space one multiplies (5.7) by the difference of the $\mathrm{H}$ characters of the $(s)$ and $(c)$ representations of $\mathrm{SO}(2 d)$. This difference of characters contains only factors of $\left(\mathrm{e}^{i \pi \mu \cdot \alpha}-\mathrm{e}^{-i \pi \mu \cdot \alpha}\right)$ and thus cancels all but the $\mathrm{H}$ part of the denominator of (5.7), to leave one with the following expression for the index:

$$
I_{\Lambda}(\mu)=(-1)^{d} \frac{\sum_{w \in \mathrm{W}_{\mathrm{G}}} \in(w) \mathrm{e}^{-2 \pi i \mu \cdot\left[w\left(A+\rho_{\mathrm{G}}\right)-\rho_{\mathrm{H}}\right]}}{\prod_{\alpha \in \Delta^{+}(\mathrm{H})}\left(1-\mathrm{e}^{2 \pi i \mu \cdot \alpha}\right)},
$$

where we have used $\prod_{\alpha \in t_{+}} \mathrm{e}^{i \pi \mu \cdot \alpha}=\mathrm{e}^{2 \pi i \mu \cdot\left(\rho_{\mathrm{G}}-\rho_{\mathrm{H}}\right)}$. It is now trivial to write this as a sum over irreducible $\mathbf{H}$ characters since the denominator has the correct form:

$$
I_{\Lambda}(\mu)=(-1)^{d} \sum_{w^{\prime} \in \mathrm{W}(\mathrm{G}) / \mathrm{W}(\mathrm{H})} \epsilon\left(w^{\prime}\right) \chi_{\mathbf{H}}\left(\Lambda^{\prime}\left(w^{\prime}\right)\right),
$$

where $w^{\prime}$ is a coset representative of $\mathrm{W}(\mathrm{G}) / \mathrm{W}(\mathrm{H})$ chosen in such a way that

$$
\Lambda^{\prime}\left(w^{\prime}\right)=w^{\prime}\left(\Lambda+\rho_{\mathrm{G}}\right)-\rho_{\mathbf{H}}
$$

is a highest weight of an $\mathrm{H}$ representation. Note that (5.9) gives one an explicit list of $\mathrm{H}$ representations that contribute to the index. For our present purposes we only need to observe that there are $r$ such irreducible characters in this expression. Since a zero mode of $G_{0}$ is necessarily a simultaneous zero mode of $G_{0}^{+}$and $G_{0}^{-}$, it follows that the total number of such zero modes is at least $r$. The fact that there are precisely $r$ zero modes has been shown in ref. [29]. This means that in the computation of the $\mathrm{H}$-character valued Euler characteristic, there are no pairs of $\mathrm{H}$ representations that contribute with opposite signs as to cancel one another. 
Moreover, each irreducible representation of $\mathbf{H}$ that contributes to the index, appears exactly once.

We can actually do better, and try solving (5.2) without restricting the solutions to those that come from the ground state of the $\mathrm{G}$ theory. If we denote the Kac-Moody algebra of $\boldsymbol{t}_{+}$by $\tilde{\boldsymbol{t}}_{+}$, then the solutions to (5.2) are in one-to-one correspondence with the (semi-infinite) cohomology elements of

$$
\mathrm{H}^{*}\left(\tilde{\boldsymbol{t}}_{+}, \Lambda\right)
$$

To find (at least some of) the non-trivial cohomology elements, we can compute the $\mathrm{H}$-character valued Euler characteristic of this Hilbert space by doing an exactly analogous calculation in the complete Kac-Moody algebra, and not merely in the zero mode sector, by using the Weyl-Kac character formula. The calculation is almost identical to the the one above, except that in the expression (5.5) one has to use the affine Weyl group, which is the semi-direct product of the finite dimensional Weyl group with the root lattice ${ }^{\star}$ [30]. This implies that the right-hand side of (5.5) gets an extra factor of $\left|\Gamma^{\mathrm{r}}(\mathrm{G}) / \Gamma^{\mathrm{r}}(\mathrm{H})\right|$, where $\Gamma^{\mathrm{r}}(\mathrm{G})$ and $\Gamma^{\mathrm{r}}(\mathrm{H})$ denote the root lattices $^{\star \star}$ of $G$ and $H$. This means that there are some contributions to the ground state of the $\mathrm{G} / \mathrm{H}$ theory that appear to come from the excited states of the $\mathrm{G}$ theory. This extra factor is, however, trivial in that it represents a multiple counting of the same physical states of the coset model. However, before showing this, we first count how many ground states we get for the $\mathrm{G} / \mathrm{H}$ theory, i.e. determine what is the dimension of the chiral ring (modulo state identifications to be discussed below).

From the index computations above it is clear that a priori we get just a lower bound on the number of elements of the chiral ring. We will show in the appendix that this is not the case and the index computation gives the full set of ground states of the Ramond sector. This non-cancellation mirrors the case for the finite dimensional groups mentioned before (similar extensions do work for the Kac-Moody case [36]).

We have seen above that for each irreducible highest weight representation of affine $\mathrm{G}$ at level $k$, we get a contribution of

$$
\hat{r}=\frac{|\mathrm{W}(\mathrm{G})|}{|\mathrm{W}(\mathrm{H})|}\left|\frac{\Gamma^{\mathrm{r}}(\mathrm{G})}{\Gamma^{\mathrm{r}}(\mathrm{H})}\right|
$$

states to the ground state of $\mathrm{G} / \mathrm{H}$ theory. Let $N_{\mathrm{G}}^{k}$ denote the total number of irreducible highest weight representations of affine $\mathrm{G}$ at level $k$. For example, if $\mathrm{G}=\mathrm{SU}(n)$, then

$$
N_{\mathrm{G}}^{k}=(k+n-1) ! /(k !(n-1) !)
$$

\footnotetext{
^ For the non-simply laced groups it is actually the lattice generated by the long roots.

$\star \star$ The root lattice of a $U(1)$ factor is defined to be lattice of its quantized momenta.
} 
Modulo taking into account the identification of states discussed below (reducing the number of inequivalent states by a factor $1 /|\mathrm{S}|$ ), it follows that the dimension $\mu$ of the chiral ring $\mathscr{R}$ is given by

$$
\mu=N_{\mathrm{G}}^{k} \cdot \frac{|\mathrm{W}(\mathrm{G})|}{|\mathrm{W}(\mathrm{H})|}\left|\frac{\Gamma^{\mathrm{r}}(\mathrm{G})}{\Gamma^{\mathrm{r}}(\mathrm{H})}\right| .
$$

In order to understand these identifications, we digress to review some general aspects of coset models (cf. ref. [31]).

In many (but not all) cases the Hilbert space of the $\mathrm{G} / \mathrm{H}$ models are obtained from pairs of representations $\Lambda$ of $\mathrm{G}$ and $\lambda$ of $\mathrm{H}$ by decomposing the former into the latter:

$$
\mathscr{H}_{\Lambda}=\sum_{\lambda} \mathscr{H}_{\Lambda \lambda} \otimes \mathscr{H}_{\lambda}
$$

The conformal theory of $\mathrm{G} / \mathrm{H}$ is then obtained by taking the Hilbert space to be

$$
\mathscr{H}_{\mathrm{G} / \mathrm{H}}=\sum \mathscr{H}_{\Lambda \lambda} \otimes \overline{\mathscr{H}}_{\Lambda \lambda},
$$

where $\overline{\mathscr{H}}$ denotes the right-moving Hilbert space. This structure for the Hilbert space is motivated by the requirement of modular invariance: if $b_{\Lambda \lambda}(\tau)$ denotes the character of $\mathscr{H}_{\Lambda \lambda}$, then it is easy to see [32] that

$$
\sum\left|b_{\Lambda \lambda}\right|^{2}
$$

is modular invariant. This description of $\mathrm{G} / \mathrm{H}$ theories is however incomplete as we will now discuss.

In general, not all subsectors of the $\mathrm{G} / \mathrm{H}$ Hilbert space $\mathscr{H}_{\Lambda \lambda}$ are inequivalent. In fact, the symmetries of the extended Dynkin diagrams of $\mathrm{G}$ and $\mathrm{H}$ will force certain identifications. At the heart of such identifications is the fact that if we have a representation $\Lambda$ of (affine) $\mathrm{G}$ at level $k$, and if we let $v$ to correspond to a weight vector of $\mathrm{G}$, which means that $\exp (2 \pi i v \cdot \mathrm{H})$ is in the center of $\mathrm{G}$, shifting the Cartan momenta by $k v$ will give a new representation of affine $\mathrm{G}$ at level $k$ which we denote by $v(\Lambda)$. (This fact is well known to mathematicians [33], and is discussed in the physics literature [34].) This is easy to understand if we recall that twisting a Kac-Moody algebra by $\exp (2 \pi i v \cdot \mathrm{H})$, where $\mathrm{H}$ denotes the Cartan currents, acts trivially on the roots, and therefore leaves the Kac-Moody algebra unbroken. Therefore, the twisted sector which is obtained from the original representation by shifting the Cartan momenta by $k v$ furnishes another representation of the affine $G$ at the same level. This is in fact very similar to the spectral flow that we discussed for the $N=2$ algebra, where we twisted the U(1) current. If we twist with a parameter $\theta$ we twist the currents of the Kac-Moody algebra and Virasoro 
algebra according to

$$
\begin{aligned}
& J_{n}^{a} \rightarrow J_{n+\theta v \cdot a}^{a}, \\
& H_{n}^{i} \rightarrow H_{n}^{i}+k \theta v^{i} \delta_{n, 0}, \\
& L_{n} \rightarrow L_{n}+\theta v \cdot H_{n}+k(\theta v)^{2} / 2 \delta_{n, 0},
\end{aligned}
$$

where $J^{a}$ denotes the currents coming from the roots, and $H^{i}$ denotes the Cartan currents. For $\theta=1$, we see that the moding of the currents is again integral, and we obtain a new representation of affine $G$ which is related to the one we started with by a symmetry of the extended Dynkin diagram. This in fact explains why the Dynkin diagram has the center of $\mathrm{G}$ as a symmetry. For example, for the $\mathrm{SU}(n)$ Kac-Moody algebra this symmetry is simply the $Z_{n}$ rotation symmetry of the extended Dynkin diagram. This shift is familiar from the study of level one simply laced groups where the Hilbert space of different representations differ only by a shift of the lattice by a weight vector.

Suppose we have a $\mathrm{G} / \mathrm{H}$ coset model and that $\mathrm{G}$ and $\mathrm{H}$ have a common center. We can use the foregoing spectral flow to probe some aspects of the $\mathrm{G} / \mathrm{H}$ models [35]. First we note that the existence of a common center implies selection rules on which pairs $(\Lambda, \lambda)$ will appear, because $\Lambda$ and $\lambda$ should transform the same way under the common center (similar to the selection rules for decomposing representations of ordinary Lie groups into representation of its subgroups). Secondly, by considering a spectral flow on $\mathrm{G}$ and $\mathrm{H}$ simultaneously generated by the common center, we see that decomposing a representation $\Lambda$ of $\mathrm{G}$ and considering the multiplicities of $\lambda$ representation of $H$, is the same problem as decomposing the $v(\Lambda)$ representation of $\mathrm{G}$ and considering the multiplicities of $v(\lambda)$ representation of $\mathrm{H}$. This implies, in particular, that

$$
\mathscr{H}_{\Lambda \lambda}=\mathscr{H}_{v(\Lambda) v(\lambda)}
$$

The identification (5.13) implies in particular that (5.11) is not a good definition of the Hilbert space of a conformal theory, as for instance the vacuum appears with multiplicity more than one, due to (5.13). One could circumvent this by dividing the Hilbert space by an overall multiplicity to avoid repetitions. This would still be consistent with the requirement of modular invariance (5.12). However, if (5.13) has fixed points (i.e. $(v(\Lambda), v(\lambda))=(\Lambda, \lambda))$, this would not be possible, because not all $\mathscr{H}_{\Lambda \lambda}$ will appear with the same multiplicity. If we take one representative Hilbert space for each orbit of the action of the common center, we will in these cases destroy modular invariance (5.12). In such cases the subsectors that are fixed under the action of the common center are not irreducible representations of the $\mathrm{G} / \mathrm{H}$ theory [35]. Finding the irreducible characters that make up the character of the 
fixed point Hilbert space is a question which has not been resolved yet, and is of fundamental importance for a better understanding of $\mathrm{G} / \mathrm{H}$ theories. In this paper we will concentrate on the case where there are no fixed points of the common spectral flow. We intend to study the fixed point cases in ref. [8].

After this long digression, we return to the consideration of the models of Kazama and Suzuki. First we should note that the $G$ in the preceding discussion should be replaced by $\mathrm{G} \times \mathrm{SO}(2 d)$. Secondly, it is important to recall that in the construction of ref. [7] the subgroup $\mathrm{H}$ is in fact embedded diagonally into $\mathrm{G} \times \mathrm{SO}(2 d)$, and therefore if we wish to generate a pure $\mathrm{H}$ shift from a given weight $v$ of $\mathrm{G}$ then $v$ must always be paired with some weight vector $\tilde{v}$ of $\mathrm{SO}(2 d)$ in such a way that a pair $(v, \tilde{v})$ generates a flow that is orthogonal to the superconformal $\mathrm{G} / \mathrm{H}$ theory. Let $\Lambda$ denote a weight of a level $k$ representation of the Kac-Moody algebra of $\mathrm{G}$, let $\tilde{\Lambda}$ be some weight of a level one Kac-Moody representation of $\mathrm{SO}(2 d)$, and let $\lambda$ be some $\mathrm{H}$ weight in the affine $\mathrm{H}$ decomposition of the product of the two foregoing representations, then $v$ generates a flow of the form

$$
\begin{aligned}
\Lambda & \rightarrow \Lambda+k \theta v, \\
\lambda & \rightarrow \lambda+\left(k+g_{\mathrm{G}}-g_{\mathrm{H}}\right) \theta v, \\
\tilde{\Lambda}_{\beta} & \rightarrow \tilde{\Lambda}_{\beta}+\theta(\beta \cdot w),
\end{aligned}
$$

where $\tilde{\Lambda}_{\beta}$ are the components of $\tilde{\Lambda}$ in the Cartan subalgebra defined by $\psi^{-\beta} \psi^{\beta}$. In other words, $\tilde{w}_{\beta}=(\beta \cdot w)$. In (5.14) we have adopted the convention that $g_{\mathrm{H}}=0$ for the $\mathrm{U}(1)$ pieces of $\mathrm{H}$. One should note that $\tilde{w}_{\beta}$ is always an integer, and thus $\tilde{w}$ is either a vector weight or a root of $\operatorname{SO}(2 d)$. This means that the flow always sends the Ramond sector to the Ramond sector and the Neveu-Schwarz sector to the Neveu-Schwarz sector. It will change the conjugacy classes when $\sum_{\beta \in t_{+}} \beta \cdot w$ is odd.

Let $S$ denote the finite symmetry group of spectral flows which acts on the $\oplus \mathscr{H}_{\Lambda \lambda}$. It is not too difficult to see that

$$
S=\frac{\Gamma^{\mathrm{w}}(\mathrm{G})}{\Gamma^{\mathrm{r}}(\mathrm{H})},
$$

where $\Gamma^{\mathrm{w}}(\mathrm{G})$ denotes the dual to the root lattice of $\mathrm{G}$, which is the weight lattice of $\mathrm{G}$ for simply laced groups. This expression for $\mathrm{S}$ can be understood as follows: each element in $\Gamma^{\mathrm{w}}(\mathrm{G})$ can be used to define a spectral flow which identifies states, and the spectral flow is trivial precisely if the element chosen happens to be on $\Gamma^{\mathrm{r}}(\mathrm{H})$ [which is therefore also in $\Gamma^{\mathrm{r}}(\mathrm{G})$ ]. Let us assume that $\mathrm{S}$ has no fixed points when it acts on the labels of $\mathrm{G} / \mathrm{H}$ theory. Then the number of inequivalent chiral primary states that have identified so far is given by $(5.10)$ divided by $|S|$, which is therefore equal to

$$
\mu=\frac{1}{|\mathrm{Z}(\mathrm{G})|}\left[N_{\mathrm{G}}^{k}\right] \frac{|\mathrm{W}(\mathrm{G})|}{|\mathrm{W}(\mathrm{H})|},
$$

where $Z(G)$ denotes the center of $G$. 
There is another way to understand the counting (5.15). This is based on the semiclassical analysis: if we just restrict to states in the ground state of $G$ which gives rise to ground states of $\mathrm{G} / \mathrm{H}$, each of the $N_{\mathrm{G}}^{k}$ representations of affine $\mathrm{G}$ contributes $|W(G)| /|W(G)|$, but the center of $G$ causes identification on this space by a group $Z(G)$, which reproduces the counting (5.15). Moreover, we can use this, together with the knowledge of which $\mathrm{H}$ representations appear (given explicitly by (5.9)) to compute the Poincaré polynomial for all the models of ref. [7] (see ref. [8]).

As an example consider the supersymmetric grassmannian models

$$
\frac{\mathrm{SU}(m+n)}{\mathrm{SU}(m) \times \mathrm{SU}(n) \times \mathrm{U}(1)},
$$

where $\mathrm{SU}(m+n)$ has level $k$. Then one can show that the $\mathrm{U}(1)$ lattice has spacing ${ }^{\star}$

$$
\Delta q=(k+m+n)(m+n) m n,
$$

where $q$ is the $\mathrm{U}(1)$ charge defined with the normalization of ref. [7]. For this theory there are two basic weights that generate the common center: (i) a weight $v_{1}$ of the fundamental of $\mathrm{SU}(m+n)$ which, when projected onto $\mathrm{H}$ is a weight of the fundamental of $\mathrm{SU}(m)$ and a singlet of $\mathrm{SU}(n)$; and (ii) a fundamental weight $v_{2}$ of $\mathrm{SU}(m+n)$ which yields a singlet of $\mathrm{SU}(m)$ and the complex conjugate of a fundamental weight of $\mathrm{SU}(n)$. The weights $v_{1}$ and $v_{2}$ generate transformations that both cyclically permute, one step counterclockwise, the extended Dynkin labels attached to the extended Dynkin diagram of $\mathrm{SU}(m+n)$. The transformation $v_{1}$ fixes the $\mathrm{SU}(n)$ extended Dynkin labels, while cyclically permuting, again by one step counterclockwise, the extended $\mathrm{SU}(m)$ labels. The transformation $v_{2}$ fixes $\mathrm{SU}(m)$ and cycles $\mathrm{SU}(n)$. From the second equation of (5.14) one can also see that $v_{1}$ and $v_{2}$ change the $\mathrm{U}(1)$ charge by

$$
\begin{aligned}
& \Delta_{1} q=(k+m+n) n, \\
& \Delta_{2} q=-(k+m+n) m .
\end{aligned}
$$

In addition, $v_{1}$ (respectively, $v_{2}$ ) flips the conjugacy classes of $\operatorname{SO}(2 d)$ when $n$ (respectively, $m$ ) is odd. It is elementary to see that $v_{1}$ has order $(m+n) m$ and $v_{2}$ has order $(m+n) n^{\star \star}$, and that they generate an abelian group of order $(m+n) m n$. It is straightforward to see that if $k, m$ and $n$ have no overall common factor then

\footnotetext{
* If $m$ and $n$ have a common divisor $(m, n)$ one can reduce $\Delta q$ by $(m, n)$. This is optional, because we will ultimately mod out by all the symmetries in $S$ so as to only count inequivalent states. This modding out will automatically include any necessary reduction in $\Delta q$.

$\star \star$ Again note that if $m, n$ have common factors, by reducing the periodicity of $\mathrm{U}(1)$ charge by $(m, n)$, the order of these elements will be reduced by $(m, n)$.
} 
none of the transformations $v$ in this group have a fixed point when acting on the Hilbert spaces as in (5.13). As a result, in these circumstances one could then define the $\mathrm{G} / \mathrm{H}$ theory to be the one whose Hilbert space consists of exactly one $\mathscr{H}_{\Lambda \lambda}$ from each orbit of the spectral flow group action. This theory has only one vacuum, and its partition function is certainly modular invariant as it is simply (5.12) divided by $(m+n) m n$.

As explained above, the common center yields selection rules for the labels of the characters of the superconformal $\mathrm{G} / \mathrm{H}$ models. In the foregoing example, the characters will be labelled by an $\mathrm{SU}(m+n)$ weight $\Lambda$, an $\mathrm{SU}(m)$ weight $\lambda_{1}$, an SU $(n)$ weight $\lambda_{2}$ and the U(1) charge $q$. Let $n_{j}, j=1, \ldots,(m+n-1)$ be the Dynkin labels of $\Lambda$, and let $p_{j}, j=1, \ldots,(m-1)$ and $r_{j}, j=1, \ldots,(n-1)$ be the Dynkin labels of $\lambda_{1}$ and $\lambda_{2}$ respectively. Since the representation of $\operatorname{SU}(m+n)$ is level $k$, the labels $n_{j}$ must satisfy $\sum n_{j} \leqslant k$, while $p_{j}$ and $r_{j}$ satisfy $\sum p_{j} \leqslant k+n$ and $\sum r_{j} \leqslant k+m$. In addition to the fact that $q$ is identified modulo the lattice spacing (5.16), the selection rules also show that we must have

$$
\begin{aligned}
& q \equiv-m \sum_{j=1}^{m+n-1}\left(j n_{j}\right)+(m+n) \sum_{j=1}^{m-1}\left(j p_{j}\right)+\epsilon \frac{1}{2} m n(m+n) \quad \bmod m(m+n), \\
& q \equiv n \sum_{j=1}^{m+n-1}\left(j n_{j}\right)-(m+n) \sum_{j=1}^{n-1}\left(j r_{j}\right)+\epsilon \frac{1}{2} m n(m+n) \quad \bmod n(m+n),
\end{aligned}
$$

where $\epsilon=1$ in the Ramond sector of the fermions, and $\epsilon=0$ in the Neveu-Schwarz sector.

The dimension of the chiral ring $\mathscr{R}$ for the grassmannian example is easily computed from (5.15) to be

$$
r=(n+m+k-1) ! /(n ! m ! k !)
$$

(note that this is an integer because $m, n, k$ have no common divisors). Similar formulas could be worked out using (5.15) for the other models of Kazama and Suzuki.

Now we wish to connect some of the Kazama-Suzuki models with LandauGinzburg models. Consider the simplest cases of Kazama-Suzuki models, that is, the HSS cases. In particular, let us restrict our attention to the subset characterized by simply laced groups G. We begin by considering the simplest HSS models: those with level one. It is easily seen that the spectral flow has no fixed points for level $k=1$ HSS simply laced models. The dimension of the chiral primary ring is easily obtained from (5.15), and is simply

$$
\mu=|\mathrm{W}(\mathrm{G})| /|\mathrm{W}(\mathrm{H})|
$$


To identify the chiral primary states it is easy to see, using spectral flow, that we can always choose the affine representation of $G$ to be the basic one (corresponding to the trivial representation of $\mathrm{G}$ in the ground state). In the truncated theory, i.e. the "semi-classical" analysis, in the Ramond sector the Hilbert space will be simply $\wedge \boldsymbol{t}_{+}$as discussed before. The operator $G^{+}$vanishes identically on this Hilbert space (as $J^{\alpha}=0$ and $f_{\alpha \beta}^{\gamma}=0$ ). Therefore all we have to do to find the elements in the $\mathrm{G} / \mathrm{H}$ ground state is to find the irreducible $\mathrm{H}$ representations of $\wedge \boldsymbol{t}_{+}$for which we know there are $|\mathrm{W}(\mathrm{G}) / \mathrm{W}(\mathrm{H})|$ in number. One way to describe the $\mathrm{H}$ irreducible reps, is to enlarge the Hilbert space by including the right-mover Hilbert space involving $\bar{\psi}^{-\alpha}$. These fermions could be thought of as the anti-holomorphic tangent vectors to $\mathrm{G} / \mathrm{H}$. Then the elements of the $\mathrm{G} / \mathrm{H}$ theory ground state would be in one-to-one correspondence with the $\mathrm{H}$ singlets of

$$
\mathscr{H}_{\mathrm{G} / \mathbf{H}}=\left.\left(\wedge \boldsymbol{t}_{+} \wedge \overline{\boldsymbol{t}}_{-}\right)\right|_{\mathrm{H} \text { singlet }},
$$

because this counts exactly the number of $H$ irreps of $\wedge \boldsymbol{t}_{+}$. However, for $\mathrm{G} / \mathrm{H}$ being a symmetric space, the right-hand side of (5.18) is identical to the cohomology of $\mathrm{G} / \mathrm{H}$ itself (this result is due to Cartan, and a proof of it is a simple extension of an argument for the cohomology of groups; see for example [37]). Moreover, the elements in (5.18) obviously inherit a ring structure from wedging the forms, and that is in fact identical to the ring structure of $H^{*}(\mathrm{G} / \mathrm{H})$. In other words, the equality between $\mathrm{H}^{*}$ and (5.18) respects the ring structures as well. The cohomology of Kähler manifolds $\mathrm{G} / \mathrm{H}$ is concentrated in the diagonal of the Hodge diamond (i.e., $b_{p . q}=0$ unless $p=q$ ). Using the form of the current (5.4) we therefore see that for each element in the cohomology of $\mathrm{G} / \mathrm{H}$ of dimension $(p, p)$ we get a chiral primary field of charge $(p, p)(g+1)$. However, as in sect. 3 , it is not necessarily true that this ring, though isomorphic to the cohomology ring of $\mathrm{G} / \mathrm{H}$, is isomorphic to the ring $\mathscr{R}$ of the full, untruncated two dimensional theory. From the foregoing discussion it is clear that $\mathscr{R}$ will be at least a deformation of the cohomology ring. We will return to the relation between $\mathscr{R}$ and the cohomology ring below.

It is not too difficult to identify these theories (HSS simply laced level one) with Landau-Ginzburg theories. We give the set of generators of primary fields and their weights in table 1. It is straightfoward to check that the relation (4.7) is indeed satisfied. To prove that a given coset model is indeed a Landau-Ginzburg model, one would have to obtain the superpotential $W$ which determines the ring structure by (4.3). This might be possible by arguments along the lines indicated in the previous section, that is, by identifying $W$ with $G^{+} \bar{G}^{+}$. At any rate, $W$ can in principle be found by studying the operator products of chiral primary fields in the $\mathrm{G} / \mathrm{H}$ model.

We now return to the question of whether these rings are isomorphic to the $\mathrm{G} / \mathrm{H}$ cohomology rings. If this is so, then the cohomology ring of $\mathrm{G} / \mathrm{H}$ must itself have 
TABLE 1

Charges of the Landau-Ginzburg fields for the simply laced HSS coset models at level $k=1$.

The charge unit is always the denominator appearing in the expression for $c$.

\begin{tabular}{|c|c|c|}
\hline Coset theory & $c$ & Degrees of generators \\
\hline $\mathrm{SU}(n+m)$ & $3 n m$ & \multirow{2}{*}{$1,2 \ldots, \min (n, m)$} \\
\hline$\overline{\mathrm{SU}(n) \otimes \mathrm{SU}(m) \otimes \mathrm{U}(1)}$ & $\overline{m+n+1}$ & \\
\hline $\operatorname{SO}(n+2)$ & $3 n$ & $1 n$ \\
\hline$\overline{\mathrm{SO}(n) \otimes \mathrm{U}(1)},(n$ even $)$ & $\overline{n+1}$ & $1, n / 2$ \\
\hline $\mathrm{SO}(2 n)$ & $3 n(n-1)$ & $\int i=1, \ldots, n / 2$ ( $n$ even $)$ \\
\hline$\overline{\mathrm{SU}(n) \otimes \mathrm{U}(1)}$ & $\overline{2(2 n-2+1)}$ & $4 t-2, \quad\{i=1, \ldots,(n-1) / 2(n$ odd $)$ \\
\hline$E_{6}$ & 48 & 1 \\
\hline$\overline{\mathrm{SO}(10) \otimes U(1)}$ & $\overline{13}$ & 1,4 \\
\hline $\mathrm{E}_{7}$ & 81 & 150 \\
\hline $\mathrm{E}_{6} \otimes \mathrm{U}(1)$ & $\overline{19}$ & $1,5,9$ \\
\hline
\end{tabular}

the structure (4.3). As can be checked case by case, this is indeed true for the HSS simply laced manifolds! (We have however not yet checked the case where $\mathrm{G}=\mathrm{E}_{6}$ or $E_{7}$; see ref. [8].) Even though the structure of the cohomology rings for the $\mathrm{G} / \mathrm{H}$ manifolds is well-known to mathematicians [38], it had not been shown that for simply laced HSS the ring $\mathrm{H}^{*}(\mathrm{G} / \mathrm{H})$ can be expressed in the form (4.3). The cohomology rings of general kählerian $\mathrm{G} / \mathrm{H}$ manifolds cannot always be expressed in this form. For example the cohomology ring of $\mathrm{SU}(3) / \mathrm{U}(1) \times \mathrm{U}(1)$ is not of the form (4.3).

The fact that for the HSS simply laced spaces the cohomology ring is of the form $\mathrm{C}\left[x_{i}\right] / \mathrm{d} W\left(x_{i}\right)$ does not prove that the superpotential $W$ of a given LandauGinzburg model is identical to the function $W$ that generates the cohomology ring of $\mathrm{G} / \mathrm{H}$. However, in some cases this can be shown to be true and we conjecture that it is generally true. For example, it is true for all the HSS manifolds whose $g+1$ cohomology vanishes (that is, $b_{g+1}=0$ ). These cases correspond to the conformal theories that have no massless moduli, and thus $\mathscr{R}$ can readily be made identical to the cohomology ring of $\mathrm{G} / \mathrm{H}$ by a field redefinition. For example, the $\mathrm{SU}(n+1) / \mathrm{U}(n)=C P^{n}$ superconformal model has the chiral ring defined by

$$
\mathscr{R}=\left\{1, x, x^{2}, \ldots \text { subject to } x^{n+1}=0\right\}
$$

This is exactly the definition of the cohomology ring of $C P^{n}$ where $x$ corresponds to the generator with degree $(1,1)$. The ring is that of $\mathrm{A}_{n+1}$ singularity with $W=x^{n+2}$, and corresponds to a minimal $N=2$ model. A further example where the isomorphism of $\mathscr{R}$ with the cohomology ring of $\mathrm{G} / \mathrm{H}$ is obvious is $\mathrm{SU}(4) / \mathrm{SU}(2) \times \mathrm{SU}(2)$ 
$\times \mathrm{U}(1)$. In this case $W$ can be chosen to be

$$
W=x_{1}^{5}-5 x_{1}^{3} x_{2}+5 x_{1} x_{2}^{2},
$$

where the charges of $x_{1}, x_{2}$ are $1 / 5$ and $2 / 5$, respectively. Another case is $\mathrm{SO}(n+2) / \mathrm{SO}(n) \times \mathrm{SO}(2)$ (with $n$ even), for which the superpotential is

$$
W=x_{1}^{n+1}+x_{1} x_{2}^{2} .
$$

This corresponds to minimal $N=2$ models of type $D_{n+2}$.

One of the simplest cases where it is non-trivial to find the exact form of $W$ is the model $\mathrm{SU}(5) / \mathrm{SU}(3) \times \mathrm{SU}(2) \times \mathrm{U}(1)$. In this case the function that generates the cohomology ring is given by

$$
W=x_{1}^{6}-6 x_{1}^{4} x_{2}+9 x_{1}^{2} x_{2}^{2}-2 x_{2}^{3},
$$

the degrees of $x_{1}, x_{2}$ being $1 / 6$ and $1 / 3$, respectively. The superpotential for the corresponding Landau-Ginzburg theory could differ from this by the addition of a massless modulus:

$$
W \rightarrow W+a x_{2}^{3} .
$$

(That there is only one massless modulus can be seen by considering the Poincaré polynomial: $b_{6}=1$; put differently, though there are four monomials of degree one, three possible changes of variables corresponding to rescaling $x_{1}$ and $x_{2}$ and to $x_{2} \rightarrow x_{2}+b x_{1}^{2}$ leave only one monomial as modulus.) One way to find whether $a$ is zero or not is to use the OPE in the coset model. This is not a trivial computation. However, we use a different strategy in this case. The reason is that this model is exactly solvable for all values of $a$, and corresponds to a $Z_{6}$ orbifold of a two dimensional torus [2,3]. For $a=0$ one easily finds that the left-momenta of the torus is given by $P_{\mathrm{L}}=(1 / \sqrt{3})\left(n_{1}+n_{2} \omega\right)$, where $\omega$ is a third root of unity, and $n_{1}$ and $n_{2}$ are integers. The conformal dimensions of the $Z_{6}$ orbifold of this model can be easily computed and is found to agree with the coset model spectrum. This proves that $a=0$ and in this non-trivial case the $W$ which generates the cohomology ring of the coset manifold is isomorphic to the superpotential for the conformal theory.

As an aside, we note that (see ref. [8] for details) that the cohomology of $\mathrm{SU}(m+n) / \mathrm{SU}(m) \times \mathrm{SU}(n) \times \mathrm{U}(1)$ can be generated by $m$ elements $x_{1}, \ldots, x_{m}$, and $W=z_{1}^{n+m+1}+\cdots+z_{n}^{n+m+1}$, where $W$ is to be expressed in terms of $x_{i}$ using the relation that $x_{i}$ are elementary symmetric polynomials of $z_{l}$, i.e. ${ }^{\star}$

$$
x_{i}=\sum_{1 \leqslant l_{1}<l_{2}<\cdots<l_{i} \leqslant n} z_{l_{1}} z_{l_{2}} \ldots z_{l_{i}} \text {. }
$$

* This form for $W$ was developed during conversations with J. Bernstein and simplifies an earlier description of $W$ that we had obtained. 
The superpotential $W$ for the superconformal model on $\mathrm{SU}(m+n) / \mathrm{SU}(m) \times$ $\mathrm{SU}(n) \times \mathrm{U}(1)$, if not exactly equal to the above function, will in general be a deformation of it by the addition of massless moduli.

So far we have been concentrating on the simply laced HSS models at level one. Before leaving the level one case, it will be useful for us to make the following observation: for the HSS models, the Dynkin diagram of the group $\mathrm{H}$ can be obtained by deleting one of the nodes (with Dynkin number one) of the group $\mathrm{G}$ and replacing it by the $U(1)$. Now, consider the representation $\Xi$ of $G$ which corresponds to putting a one on this node, and zero elsewhere. This representation has the same dimension as the cohomology ring of $\mathrm{G} / \mathrm{H}$. Moreover, if we choose $\rho_{\mathrm{G}}$ to be half of the sum of positive roots of $\mathrm{G}$, the grading of $\Xi$ with respect to the $\mathrm{U}(1)$ charge $\rho_{\mathrm{G}} \cdot \mathrm{H}$ is isomorphic to the grading of the cohomology of $\mathrm{G} / \mathrm{H}$ (after an overall shift). This fact is known to mathematicians [39].

To see how this works recall that the Poincaré polynomial of the cohomology ring is defined by

$$
P(t)=\sum_{k} b_{k} t^{k}
$$

where $b_{k}=b_{k, k}$ are the Betti numbers. For any coset manifold $\mathrm{G} / \mathrm{H}$ with rank $\mathrm{G}=\operatorname{rank} \mathrm{H}=l$ (we need not assume that $\mathrm{G} / \mathrm{H}$ is a HSS), $P(t)$ is given by [40]

$$
P(t)=\prod_{j=1}^{l} \frac{\left(1-t^{d_{j}(\mathrm{G})}\right)}{\left(1-t^{d_{j}(\mathrm{H})}\right)},
$$

where $d_{j}(\mathrm{G})$ and $d_{j}(\mathrm{H})$ are the degrees of the Casimirs of $\mathrm{G}$ and $\mathrm{H}$ respectively, with the convention that for a $\mathrm{U}(1)$ factor the corresponding $d_{j}$ is equal to 1 .

On the other hand consider the character of the group element $h=t^{-\rho_{\mathrm{G}}} \cdot \mathrm{H}=$ $\mathrm{e}^{-2 \pi i \rho_{\mathrm{G}} \cdot \mathrm{H}}$ evaluated in some representation of $\mathrm{G}$ with highest weight $\Lambda$. Using the Weyl character formula one obtains:

$$
\begin{gathered}
\chi_{\Lambda}(h) \equiv \operatorname{Tr}_{\Lambda}[h]=\frac{\sum_{w \in \mathrm{W}^{\epsilon}} \epsilon(w) \mathrm{e}^{-2 \pi i \rho_{\mathrm{G}} \cdot\left[w\left(\Lambda+\rho_{\mathrm{G}}\right)-\rho_{\mathrm{G}}\right]}}{\prod_{\alpha \in \Delta+(\mathrm{G})}\left(1-\mathrm{e}^{2 \pi i \nu \alpha \cdot \rho_{\mathrm{G}}}\right)} \\
=\frac{\sum_{w \in W^{\prime}} \epsilon(w) \mathrm{e}^{-2 \pi i \nu\left[w\left(p_{\mathrm{G}}\right)-\rho_{\mathrm{G}}\right] \cdot\left[\Lambda+\rho_{\mathrm{G}}\right]}}{\prod_{\alpha \in \Delta+(\mathrm{G})}\left(1-\mathrm{e}^{2 \pi i \nu \cdot \alpha \cdot \rho_{\mathrm{G}}}\right)}=t^{-\Lambda \cdot \rho_{\mathrm{G}}} \prod_{\alpha \in \Delta^{+}(G)} \frac{\left(1-t^{\alpha \cdot(\Lambda+\rho \mathrm{G})}\right)}{\left(1-t^{\left.\alpha \cdot \rho_{\mathrm{G}}\right)}\right.} .
\end{gathered}
$$

For the representation discussed above, and for a HSS we have $\alpha \cdot \Xi=0$ when $\alpha \in \mathbf{h}$ 
and $\alpha \cdot \Xi= \pm 1$ when $\alpha \in t_{ \pm}$. Hence

$$
\chi_{\Xi}(h)=t^{-\Xi \cdot \rho_{\mathrm{G}}} \prod_{\alpha \in t_{+}} \frac{\left(1-t^{\alpha \cdot \rho_{\mathrm{G}}+1}\right)}{\left(1-t^{\alpha \cdot \rho_{\mathrm{G}}}\right)} .
$$

Consider the function

$$
P_{\mathrm{G}}(t)=\prod_{\alpha \in \Delta^{+}(\mathrm{G})} \frac{\left(1-t^{\alpha \cdot \rho_{\mathrm{G}}+1}\right)}{\left(1-t^{\alpha \cdot \rho_{\mathrm{G}}}\right)}
$$

and observe that $\chi_{\Xi}(h)=t^{-\Xi \cdot \rho_{\mathrm{G}}} P_{\mathrm{G}}(t) / P_{\mathrm{H}}(t)$. For a group $\mathrm{G}$, let $b_{j}$ be the number of positive roots such that $\alpha \cdot \rho_{\mathrm{G}}=j$. Recall that $b_{1}=l$, the rank of $\mathrm{G}$, and $b_{g^{*}-1}=1$ and $b_{j}=0$ for $j \geqslant g^{*}$ (where $g^{*}$ is the Coxeter number of G). It was shown [41] that $b_{j}-b_{j+1}=n_{j}$ where $n_{j}$ is the number of Casimirs of degree $j+1$. Using this one sees that

$$
\begin{aligned}
P_{\mathrm{G}}(t) & =\sum_{j=1}^{g^{*}-1}\left[\frac{\left(1-t^{j+1}\right)}{\left(1-t^{j}\right)}\right]^{b_{j}} \\
& =\frac{1}{(1-t)^{l}} \prod_{j=1}^{l}\left(1-t^{d_{j}(\mathrm{G})}\right) .
\end{aligned}
$$

From this and (5.19) it now follows that

$$
P(t)=t^{\Xi \cdot \rho_{\mathrm{G}}} \chi_{\bar{\Omega}}(h) .
$$

Thus, in the simply laced HSS cases we can think of the chiral primary states (shifted to the Ramond sector) as to transform according to a $G$ representation $\Xi$, with their $N=2$ charges proportional to the $\mathrm{U}(1)$ charges $\rho_{\mathrm{G}} \cdot \mathrm{H}$ of that representation. Indeed one might expect that these fields transform as a representation of $G$ as is the case for solutions of the Dirac equation on homogeneous spaces.

There is an alternative way to describe the representation $\Xi$ which suggests further generalizations: each representation of $\mathrm{H}$ gives rise to a vector bundle on $\mathrm{G} / \mathrm{H}$ of the same dimension [42]. Consider the one dimensional representation of $\mathrm{H}$ corresponding to the deleted dot (which is a representation of the U(1) piece of $\mathrm{H}$ ). This gives rise to a line bundle $\mathscr{L}$ on $\mathrm{G} / \mathrm{H}$ whose holomorphic sections, by the Borel-Weil-Bott theorem, form precisely the G representation $\Xi$ we discussed above. This line bundle has a first Chern class which is in fact the Kähler class of $\mathrm{G} / \mathrm{H}$ (for the HSS, $b_{1,1}=1$ ). This in fact has the smell of geometric quantization, and suggests that there may be a way to quantize the primary chiral fields in this theory, in such a way that the phase space is $\mathrm{G} / \mathrm{H}$ itself, and the Chern class under discussion is the symplectic form for the corresponding quantization. This is similar 
to what was observed [43] in quantizing the chiral (in the two-dimensional holomorphic sense) modes of WZW on a Riemann surface in terms of the phase space which was the moduli of flat connections on the Riemann surface. Here we are not dealing with chirality only in the two-dimensional holomorphic sense, but also in the supersymmetric sense (i.e. in the sense of complex structure of $G / H$ ). Nevertheless the structure is so similar that one is tempted to conjecture that there is a geometric quantization for primary chiral fields of $\mathrm{G} / \mathrm{H}$ theory.

From these observations, we can easily make a guess for what the primary chiral fields should be for arbitrary level $k$ for HSS: simply put a $k$ on the deleted node and zero elsewhere. This defines a $G$ representation $\Xi_{(k)}$, whose highest weight is given by $k$ times the highest weight of $\Xi$. Our guess is therefore that the ground states in the Ramond sector of the $\mathrm{G} / \mathrm{H}$ model at level $k$ are given by $\Xi_{(k)}$, with $\mathrm{U}(1)$ charges proportional to $\rho_{\mathrm{G}}$. That is, we expect that the Poincare polynomial is proportional to $\chi_{\Xi_{(k)}}$, and in particular that $\operatorname{dim} \mathscr{R}=\operatorname{dim} \Xi_{(k)}$.

The motivation to put a $k$ in front of the node comes from the observation that the representation $\Xi_{(k)}$ corresponds to the holomorphic sections of $\mathscr{L}^{\otimes k}$. This has a Chern class $k$ times the $k=1$ case. This is precisely the structure of quantization observed in ref. [43], where for finding the chiral fields for level $k$, one takes the $k$-fold tensor product of the line bundle which quantizes the $k=1$ case and finds its holomorphic sections.

Now we come to the tough question: is our guess correct? Before comparing these guessed Poincaré polynomials with those that describe the spectra of the coset models, one can check that they do have the structure (4.4). More striking is the fact that for appropriate choices of generators, which follows from the Poincare polynomial, the relation (4.7) indeed reproduces the correct central charges. This is a very non-trivial check and at first sight suggests that the HSS theories, for all levels, are Landau-Ginzburg models. The number of Landau-Ginzburg fields (generators) suggested by this picture is equal to $d$, the complex dimension of $\mathrm{G} / \mathrm{H}$. We have tabulated the Poincaré polynomials in question in table 2 . We included there also the non-simply laced cases, as the central charges come out correct also for these cases.

Now we check whether these actually come from the superconformal models of ref. [7]. There is one series with higher $k$ which is easily seen to agree with our guess: It corresponds to $\mathrm{SU}(n+1) / \mathrm{SU}(n) \times \mathrm{U}(1)$ at level $k$ (this model has been independently considered in [23]) ${ }^{\star}$. Using (4.17) we see that $\operatorname{dim} \mathscr{R}$ is given by

$$
\mu=(n+k) ! /(n ! k !)
$$

which is indeed the same as the dimension of the $k$-fold symmetric tensor product

* These models are related by a duality noted in ref. [7] to $\mathrm{SU}(n+k) / \mathrm{SU}(n) \times \mathrm{SU}(k) \times \mathrm{U}(1)$ at level one, which we discussed before. 
TABLE 2

Poincaré polynomials obtained as $U(1)$ characters from $\Xi_{(k)}$. They reproduce the correct central charges. Above, $d$ denotes the denominator appearing in the corresponding expression for $c$.

\begin{tabular}{ccc}
\hline Coset theory & $c$ & Poincaré polynomial \\
\hline $\begin{array}{c}\mathrm{SU}(n) \otimes \mathrm{SU}(m) \otimes \mathrm{U}(1) \\
\mathrm{SO}(n+2)_{k}\end{array}$ & $\frac{3 k n m}{m+n+k}$ & $\prod_{i=1}^{m} \prod_{j=1}^{n} \frac{\left(1-t^{d-(i+j-1)}\right)}{\left(1-t^{(i+j-1)}\right)}$ \\
$\frac{\mathrm{SO}(n) \otimes \mathrm{U}(1)}{\left(1-t^{d-n / 2}\right)}$ & $\frac{3 k n}{\left(1-t^{n / 2}\right)} \prod_{i=1}^{n-1} \frac{\left(1-t^{d-i}\right)}{\left(1-t^{i}\right)}$ \\
$\frac{\mathrm{SO}(2 n)_{k}}{\mathrm{SU}(n) \otimes \mathrm{U}(1)}$ & $\frac{3 k n(n-1)}{2(2 n-2+k)}$ & $\prod_{i=1}^{n-1} \prod_{j=i}^{n-1} \frac{\left(1-t^{d-2(i+j-1)}\right)}{\left(1-t^{2(i+j-1)}\right)}$ \\
$\frac{\mathrm{Sp}(2 n)_{k}}{\mathrm{SU}(n) \otimes \mathrm{U}(1)}$ & $\frac{3 k n(n+1)}{2(k+n+1)}$ & $\prod_{i=1}^{n} \prod_{j=i}^{n} \frac{\left(1-t^{d-(i+j)}\right)}{\left(1-t^{(i+j)}\right)}$ \\
$\frac{\left(\mathrm{E}_{6}\right)_{k}}{\mathrm{SO}(10) \otimes \mathrm{U}(1)}$ & $\frac{48 k}{k+12}$ & $\prod_{i=1}^{11} \frac{\left(1-t^{d-i}\right)}{\left(1-t^{i}\right)} \prod_{j=4}^{8} \frac{\left(1-t^{d-j}\right)}{\left(1-t^{\prime}\right)}$ \\
$\frac{\left(\mathrm{E}_{7}\right)_{k}}{\mathrm{E}_{6} \otimes \mathrm{U}(1)}$ & $\frac{81 k}{k+18}$ & $\frac{\left(1-t^{d-9}\right)}{\left(1-t^{9}\right)} \prod_{i=1}^{17} \frac{\left(1-t^{d-i}\right)}{\left(1-t^{i}\right)} \prod_{j=5}^{13} \frac{\left(1-t^{d-j}\right)}{\left(1-t^{j}\right)}$ \\
\hline
\end{tabular}

of the fundamental representation of $\mathrm{SU}(n+1)$. Moreover, one can check that the $\mathrm{U}(1)$ charges of the $N=2$ algebra are proportional to the action of $\rho_{\mathrm{G}} \cdot \mathrm{H}$ on this representation.

How about the other coset models? We first have to check whether the number of chiral primary states in a given theory is equal to $\operatorname{dim} \Xi_{(k)}$. It turns out that the guessed dimension is in general bigger than (5.15) which was found to be the dimension of chiral primary states in a coset model. For example, if we consider $\mathrm{SU}(5) / \mathrm{SU}(3) \times \mathrm{SU}(2) \times \mathrm{U}(1)$ at level 2 , the index computation suggests 30 states, and the dimension that we guessed suggests 50 states [corresponding to $(0200)$ representation of $\mathrm{SU}(5)]$. Moreover, by explicit computation one can show that there are only 30 chiral primary states and the Poincare polynomial in this case is given by

$$
P(t)=1+2 t^{2}+2 t^{3}+4 t^{4}+3 t^{5}+6 t^{6}+3 t^{7}+4 t^{8}+2 t^{9}+2 t^{10}+t^{12} .
$$

It is easy to see that this does not correspond to a Landau-Ginzburg theory (i.e. it cannot be of the form (4.4)). This gives an example of a left-right symmetric $N=2$ model which has primary chiral ring elements with $q_{\mathrm{L}}=q_{\mathrm{R}}$, and yet it is not a Landa!" Ginzburg theory.

It is surprising that most Kazama-Suzuki models as they stand do not correspond to Landau-Ginzburg models. However, in view of the fact that our guessed 
Poincaré polynomials seem to describe consistent Landau-Ginzburg models with central charges equal to those of the coset models, we are tempted to conjecture that there should exist modifications of the coset models (such as their orbifolds) which lead to agreement with our conjecture and which do not correspond to Landau-Ginzburg models. We will discuss these issues further in ref. [8].

At any rate, it would be nice if one can give a theoretical interpretation to the ring structures that appear in coset models at arbitrary $k$, similar to the case $k=1$ for simply laced HSS. It could be that for each $k$, there is a manifold $\mathrm{M}_{k}$, whose cohomology ring has the same gradation as that of the conformal model, and that its ring structure is equal to, or a deformation of, that of the conformal model. The manifold $\mathbf{M}_{k}$ is presumably some manifold embedded in the loop space of $\mathrm{G} / \mathrm{H}$, and it should be an interesting question to unravel its geometry in relation to the coset conformal models.

Also one might try to find a geometric quantization that counts the number of chiral primary fields in the Kazama-Suzuki models for all $k$. If such a picture exists, the computation of the dimension (5.15) of chiral rings suggests that the dimension of the corresponding symplectic manifold should be the rank of $\mathrm{G}$.

Admittedly we have condensed a lot of information in this section. This is mainly because of the combination of the rich structure of kählerian coset spaces and $N=2$ superconformal algebra. More discussion on some of the points discussed here, together with a discussion on some other aspects of KS models will appear elsewhere [8].

\section{Conclusions}

We have studied some general properties of $N=2$ superconformal models which follow entirely from the $N=2$ superconformal algebra. In particular, we have discussed the existence of certain finite (nilpotent) rings which resemble cohomology rings of manifolds (and which, in some cases, are isomorphic to them). The U(1) spectral flow of $N=2$ models was used as a powerful technique to deduce certain properties of these rings.

Moreover, we discussed $N=2$ Landau-Ginzburg models in view of these general results and gave another proof of the relation between central charge and the charges of the superfields found in ref. [1]. We also investigated under what conditions an $N=2$ model has a ring structure isomorphic to that of a Landau-Ginzburg model. We did this by identifying the superpotential with $G^{+} \bar{G}^{+}$, the chiral two-dimensional gravitino field.

We also studied some aspects of coset models with $N=2$ supersymmetry. We find that they have a rich and beautiful structure and some of them are Landau-Ginzburg models. This connection has given rise to a whole class of mathematical questions, which we believe are quite interesting in their own right. 
We would like to thank J. Bagger, J. Bernstein, R. Bott, J. Distler, M. Douglas, S. Giddings, B. Greene, M. Grisaru, J. Harris, V. Kac, B. Kostant, S. Kumar, W. Schmid, E. Shpiz, I. Singer, H. Sonoda, and E. Witten for valuable discussions. The research of W.L. is supported by the US Department of Energy under contract No. DE-AC0381-ER 40050, and that of C.V. by NSF contract PHY-87-14654, and that of N.W. is supported in part by the NSF under grant No. 87-08447, and also by a fellowship from the Alfred P. Sloan Foundation. W.L. is indebted to the Mathematics Department of MIT for hospitality.

\section{Appendix A}

It turns out that it is relatively easy to show that the index computation of sect. 5 yields all the states in the chiral ring of the $N=2$ superconformal Theory. That is, there are no cancellations in the index computation for any given G-label, $\Lambda$, and thus the total number of zero-modes of $G_{0}=G_{0}^{+}+G_{0}^{-}$is equal to the index of $G_{0}$. Moreover, given any G-label, $\Lambda$, one can obtain an explicit formula for the labels $\lambda$ of $\mathrm{H}$ that give rise to a Ramond ground state (with $L_{0}=c / 24$ ) in the $N=2$ superconformal model.

Suppose we have a highest weight state, $\Lambda$, of level $k$, affine $\mathrm{G}$, and a spinor highest weight state, $\tilde{\Lambda}$, of level one, affine $\operatorname{SO}(\operatorname{dim}(G / H))$. Suppose that the tensor product of these two representations contains a highest weight representation of affine $\mathrm{H}$ with highest weight $\lambda$, and level $k+g_{\mathrm{G}}-g_{\mathrm{H}}$. Let $n$ denote the grade at which $\lambda$ occurs in the tensor product of the affine $G$ and affine $\operatorname{SO}(\operatorname{dim}(G / H))$ representations. It follows from the work of ref. [7] that the conformal dimension of the corresponding state in the $\mathrm{G} / \mathrm{H}$ theory is

$$
\Delta=n+\frac{1}{2(k+g)}\left[|\Lambda|^{2}+2 \rho_{\mathrm{G}} \Lambda-\left(|\lambda|^{2}+2 \rho_{\mathrm{H}} \lambda\right)\right]+\frac{1}{2}|\tilde{\Lambda}|^{2},
$$

where $\lambda$ is defined so that it contains the momenta of any U(1) factors in $H$. The central charge of the corresponding theory is given by [7]

$$
c=\frac{1}{2}\left(d_{\mathrm{G}}-d_{\mathrm{H}}\right)+\frac{k}{(k+g)} d_{\mathrm{G}}-\frac{\left(k+g-g_{\mathrm{H}}\right)}{k+g} d_{\mathrm{H}},
$$

where $d_{\mathrm{G}}$ and $d_{\mathrm{H}}$ are the dimensions of $\mathrm{G}$ and $\mathrm{H}$ respectively. Let $d=\left(d_{\mathrm{G}}-d_{\mathrm{H}}\right) / 2$ denote the complex dimension of $G / H$. Using the strange formulae for $G$ and $H$ one can rewrite (A.2) as

$$
c=\frac{3}{4} d-\frac{12}{(k+g)}\left(\left|\rho_{\mathrm{G}}\right|^{2}-\left|\rho_{\mathrm{H}}\right|^{2}\right) .
$$


Finally, by observing that for the spinor highest weight of $\operatorname{SO}(\operatorname{dim}(\mathrm{G} / \mathrm{H}))$ one has $\frac{1}{2}|\tilde{\Lambda}|^{2}=\frac{1}{8} d$ one arrives at the following more succinct expression for the conformal weight of our state:

$$
\Delta-\frac{c}{24}=n+\frac{1}{2(k+g)}\left(\left|\Lambda+\rho_{\mathrm{G}}\right|^{2}-\left|\lambda+\rho_{\mathrm{H}}\right|^{2}\right) .
$$

To find the zero-modes of $G_{0}^{ \pm}$we simply have to find all $\Lambda, \lambda$ and $n$ for which (A.3) vanishes.

We can make a further simplification by introducing the complete affine labels. Recall that an affine label, $\hat{\lambda}$, corresponding to a weight $\lambda$ occurring at grade $n$ in a representation of (Kac-Moody) central charge $k$, is written $\hat{\lambda}=(\lambda, k,-n)$. The inner product of two such labels is defined by

$$
(\lambda, k, n) \cdot\left(\lambda^{\prime}, k^{\prime}, n^{\prime}\right)=\lambda \cdot \lambda^{\prime}+k n^{\prime}+n k^{\prime} .
$$

Moreover, this inner product is invariant under the action of the affine Weyl group. As usual [30], introduce the affine vectors

$$
\hat{\rho}_{\mathrm{G}}=\left(\rho_{\mathrm{G}}, g, 0\right), \quad \hat{\rho}_{\mathrm{H}}=\left(\rho_{\mathrm{H}}, g_{\mathrm{H}}, 0\right) .
$$

The fact that $\Lambda$ denotes the ground state of a level- $k$ representation of affine $\mathrm{G}$, whereas $\lambda$ defines a level $k+g-g_{\mathrm{H}}$ representation of affine $\mathrm{H}$ and appears at grade $n$ means that the corresponding labels are

$$
\hat{\Lambda}=(\Lambda, k, 0), \quad \hat{\lambda}=\left(\lambda, k+g-g_{\mathrm{H}},-n\right) .
$$

Eq. (A.3) may now be rewritten

$$
\Delta-\frac{c}{24}=\frac{1}{2(k+g)}\left[\left|\hat{\Lambda}+\hat{\rho}_{\mathrm{G}}\right|^{2}-\left|\hat{\lambda}+\hat{\rho}_{\mathrm{H}}\right|^{2}\right]
$$

where || is now the length defined via the inner product (A.4). The problem now is to consider the tensor product of the affine $G$ representation $\hat{\Lambda}$ and the affine, level-one $\operatorname{SO}(2 d)$ spinor representation $\tilde{\Lambda}$, and find the $\mathrm{H}$-weights, $\hat{\lambda}$ such that eq. (A.5) vanishes. We solve this by first recasting the $N=2$ supersymmetric $[\mathrm{G} \times \mathrm{SO}(2 d)] / \mathrm{H}$ model as a quotient of two supersymmetric models. That is, one observes that the foregoing model is manifestly isomorphic to $[\mathrm{G} \times \mathrm{SO}(\operatorname{dim} \mathrm{G})] /$ $[\mathrm{H} \times \mathrm{SO}(\operatorname{dim} \mathrm{H})]$, where $\mathrm{SO}(\operatorname{dim} \mathrm{H})$ has the obvious level-one embedding into $\mathrm{SO}(\operatorname{dim} \mathrm{G})$. Consider now the level $g_{\mathrm{H}}$ conformal embedding of $\mathrm{H}$ into $\mathrm{SO}(\operatorname{dim} \mathrm{H})$. Observe that the diagonal embedding $\mathrm{H} \rightarrow \mathrm{H} \times \mathrm{SO}(\operatorname{dim} \mathrm{G})$ induces a level $k+g$ embedding of $\mathrm{H}$ into $\mathrm{G} \times \mathrm{SO}(\operatorname{dim} G)$. Let $\tilde{\Lambda}$ now denote a spinor highest weight of $\mathrm{SO}(\operatorname{dim} \mathrm{G})$ and $\tilde{\lambda}$ denote a spinor highest weight of $\operatorname{SO}(\operatorname{dim} H)$. Observe that under 
the embedding $\mathrm{H} \rightarrow \mathrm{SO}(\operatorname{dim} H)$, the spinor weight $\tilde{\lambda}=\left(\frac{1}{2}, \frac{1}{2}, \ldots, \frac{1}{2}\right)$ corresponds to the $\mathrm{H}$-weight, $\rho_{\mathrm{H}}$. Suppose we take the tensor product of the $\hat{\Lambda}$ representation of affine $\mathrm{G}$ and the level-one $\tilde{\Lambda}$ representation of $\mathrm{SO}(\operatorname{dim} G)$ and find all the level $(k+g)$ affine $\mathrm{H}$ representations (induced via $\mathrm{H} \rightarrow \mathrm{G} \times \mathrm{SO}(\operatorname{dim} \mathrm{G})$ ) with highest weight labels $\hat{\lambda}^{\prime}=\left(\lambda^{\prime}, k+g,-n\right)$ satisfying

$$
\left|\hat{\Lambda}+\hat{\rho}_{\mathrm{G}}\right|^{2}-\left|\hat{\lambda}^{\prime}\right|^{2}=0
$$

Clearly, if $\hat{\lambda}=\left(\lambda, k+g-g_{\mathrm{H}},-n\right)$ is a weight that represents a solution to our original problem then when we tensor it with the spinor weight $\tilde{\lambda}$ and reduce the pair $(\hat{\lambda}, \tilde{\lambda})$ to a weight of $\mathrm{H}$ at level $(k+g)$, the result is $\hat{\lambda}^{\prime}=\left(\lambda+\rho_{\mathrm{H}}, k+\mathrm{g},-n\right)$ which satisfies (A.6). As a result the solutions of (A.6) contain all the solutions to the original problem.

Now consider the level- $g$ conformal embedding $G \rightarrow \operatorname{SO}(\operatorname{dim} G)$. The fact that this is a conformal embedding means that all the characters of affine, level-one $\mathrm{SO}(\operatorname{dim} \mathrm{G})$ are finitely reducible into characters of affine $\mathrm{G}$ at level $\mathrm{g}$. In particular, it is an elementary exercise in the use of the Weyl-Kac character formula to show that the spinor representation (or the sum of the two spinor representations if $\operatorname{dim} G$ is even) of $\operatorname{SO}(\operatorname{dim} G)$ decomposes into $2^{l}$ copies of the affine G-character with affine label $\hat{\rho}_{\mathrm{G}}=\left(\rho_{\mathrm{G}}, g, 0\right)$, where $l$ is the rank of $\mathrm{G}$. Thus we look for all $\mathrm{H}$-weights $\hat{\lambda}^{\prime}$ satisfying eq. (A.6) in the tensor product of the two affine $G$ representations with affine labels $\hat{\Lambda}$ and $\hat{\rho}_{\mathrm{G}}$. This tensor product has (Kac-Moody) central charge $(k+g)$, and because it is the tensor product of two affine $\mathrm{G}$ representations it is invariant under the affine Weyl group $\hat{W}(G)$ of $G$. Suppose $\hat{\lambda}^{\prime}$ is weight satisfying eq. (A.6). Then there is an element $w$ in $\hat{\mathrm{W}}(\mathrm{G})$ such that $w\left(\hat{\lambda}^{\prime}\right)$ lies in the fundamental (affine) Weyl chamber. In other words, if $\hat{\alpha}_{i}, i=0,1, \ldots, l$, are a set of simple roots for affine $\mathrm{G}$, we can arrange that

$$
w\left(\hat{\lambda}^{\prime}\right) \cdot \hat{\alpha}_{i} \geqslant 0
$$

for all $i$. Moreover $w\left(\hat{\lambda}^{\prime}\right)$ is a weight in the tensor product of affine $G$ representations described above, and so we must have

$$
\left(\hat{\Lambda}+\hat{\rho}_{\mathrm{G}}\right)-w\left(\hat{\lambda}^{\prime}\right)=\sum_{i=0}^{l} k_{i} \hat{\alpha}_{i}
$$

where the $k_{i}$ are non-negative integers. (This is true because any other weight in this tensor product must be a descendent of the product of the highest weight states $\hat{\Lambda}$ and $\hat{\rho}_{\mathrm{G}}$.) Observe that because of eq. (A.7) and the fact that $\hat{\Lambda}+\hat{\rho}_{\mathrm{G}}$ is a dominant weight, it follows that $\hat{\Lambda}+\hat{\rho}_{\mathrm{G}}+w\left(\hat{\lambda}^{\prime}\right)$ must have non-negative inner products with all the $\hat{\alpha}_{i}$. Taking the inner product of $\hat{\Lambda}+\hat{\rho}_{\mathrm{G}}+w\left(\hat{\lambda}^{\prime}\right)$ with both sides of eq. (A.8) 
one finds that

$$
\left|\hat{\Lambda}+\hat{\rho}_{\mathrm{G}}\right|^{2}-\left|w\left(\hat{\lambda}^{\prime}\right)\right|^{2} \geqslant 0
$$

with equality if and only if all the $k_{i}$ in eq. (A.8) vanish. Remembering that the inner product (A.4), and hence ||$^{2}$ are invariant under the action of $\hat{W}(G)$ it follows that we have a solution to eq. (A.6) if and only if $\hat{\lambda}^{\prime}$ can be obtained from $\hat{\Lambda}+\hat{\rho}_{\mathrm{G}}$ via the action of the affine Weyl group of $\mathrm{G}$.

Finally one should recall that, in principle, the set of solutions to eq. (A.6) is larger than the set of solutions to our original problem. However, the index calculation shows that the states that contribute to the index of $\mathrm{G}_{0}$ are precisely the $\hat{\mathrm{W}}(\mathrm{G})$ orbits of $\hat{\Lambda}+\hat{\rho}_{\mathrm{G}}$. Hence the two sets of solutions are exactly the same - there is no cancellation in the index.

It is also interesting to note that since $\hat{W}(G)=W(G) \ltimes T$, where $W(G)$ is the finite Weyl group of $G$, and $T$ are the translations by the long roots of $G$, it follows that every solution is a combination of a finite Weyl transformation of the weight $\left(\Lambda+\rho_{\mathrm{G}}\right)$ and a spectral flow by a root of $\mathrm{G}$ (and not merely a weight). Therefore, within any affine $G$ representation $\hat{\Lambda}$ all zero modes of $G_{0}$ are obtained, up to spectral flow, by finite Weyl rotations of $\Lambda+\rho_{\mathrm{G}}$. In other words, the complete set of solutions (modulo spectral flow) is obtained purely from the ground states of $G$, and thus it suffices to consider only the part of the supercurrent that is constructed from fermion and $\mathrm{Kac}-$ Moody current zero-modes, as was described in sect. 5 .

\section{References}

[1] C. Vafa and N.P. Warner, Phys. Lett. B218 (1989) 51

[2] B. Greene, C. Vafa and N.P. Warner, Calabi-Yau manifolds and renormalization group flows, Harvard preprint HUTP-88/A047

[3] E. Martinec, Algebraic geometry and effective lagrangians, Chicago preprint EFI 88-76

[4] W. Boucher, D. Friedan and A. Kent, Phys. Lett. B172 (1986) 316

[5] A. Schwimmer and N. Seiberg, Phys. Lett. B184 (1987) 191

[6] D. Friedan, A. Kent, S. Shenker and E. Witten, unpublished;

A. Sen, Nucl. Phys. B278 (1986) 289; B284 (1987) 423;

T. Banks, L.J. Dixon, D. Friedan and E. Martinec, Nucl. Phys. B299 (1988) 613

[7] Y. Kazama and H. Suzuki, New $N=2$ superconformal field theories and superstring compactification, Univ. Tokyo preprint UT-Komaba 88-8, 88-12

[8] W. Lerche, C. Vafa and N.P. Warner, in preparation

[9] E. Verlinde, Nucl. Phys. B300 (1988) 360

[10] E. Witten, Nucl. Phys. B202 (1982) 253

[11] T. Eguchi, H. Ooguri, A. Taormina and S.-K. Yang, Superconformal algebras and string compactification on manifolds with $\mathrm{SU}(n)$ holonomy, Univ. Tokyo preprint, UT-536

[12] W. Lerche and N.P. Warner, Phys. Lett. B205 (1988) 471

[13] T. Eguchi and A. Taormina, Phys. Lett. B210 (1988) 125

[14] G. Waterson. Phys. Lett. B171 (1986) 77

[15] A. Strominger and E. Witten, Commun. Math. Phys. 101 (1985) 341;

A. Strominger, Phys. Rev. Lett. 55 (1985) 2547

[16] R. Rohm and E. Witten, Ann. Phys. (New York) 170 (1986) 454

[17] D. Gepner, Scalar field theory and superstring compactification, Princeton preprint PUPT-1115 
[18] S. Hamidi and C. Vafa, Nucl. Phys. B279 (1987) 465;

L. Dixon, D. Friedan, E. Martinec and S. Shenker, Nucl. Phys. B282 (1987) 13

[19] X. Wen and E. Witten, Phys. Lett. B166 (1986) 397;

M. Dine, N. Seiberg, X.G. Wen and E. Witten, Nucl. Phys. B278 (1986) 769; B289 (1987) 319

[20] L. Dixon, Some world-sheet properties of superstring compactifications, on orbifolds and otherwise, Princeton preprint PUPT-1074

[21] J. Distler and B. Greene, Nucl. Phys. B309 (1988) 295

[22] D. Kastor, E. Martinec and S. Shenker, EFI preprint 88-31

[23] D. Gepner, Princeton preprint, PUPT-1066

[24] V.I. Arnold, Singularity theory, London Math. Lect. Notes Ser. 53 (Cambridge Univ. Press, Cambridge 1981);

V.I. Arnold, S.M. Gusein-Zade and A.N. Varchenko, Singularities of differentiable maps, vols. 1 and 2 (Birkhäuser, Basel 1985) and references therein

[25] K.S. Narain, Phys. Lett. B169 (1986) 41;

K.S. Narain, M.H. Sarmadi and E. Witten, Nucl. Phys. B279 (1986) 369

[26] L. Dixon, J. Harvey, C. Vafa and E. Witten, Nucl. Phys. B261 (1985) 678; B274 (1986) 285;

K. Narain, M. Sarmadi and C. Vafa, Nucl. Phys. B288 (1987) 551

[27] Keke Li, Phys. Lett. B219 (1989) 297

[28] C. Chevalley and S. Eilenberg. Trans. Am. Math. Soc. 63 (1948) 85

[29] B. Kostant, Ann. Math. 74 (1961) 329;

P. Griffiths and W. Schmid, Acta Math. 123 (1969) 253

[30] V.G. Kac, Infinite dimensional Lie algebras, in Prog. Math. Vol. 44 (Birkhäuser, Basel, 1983)

[31] P. Goddard, A. Kent and D. Olive, Comm. Math. Phys. 103 (1986) 105;

P. Bowcock and P. Goddard, Coset constructions and extended conformal algebras, DAMTP Cambridge preprint 1988;

M. Douglas, Ph.D. Thesis 1988

[32] V.G. Kac and M. Wakimoto, Adv. in Math. 70 (1988) 156

[33] V.G. Kac and D.H. Peterson, Adv. in Math. 53 (1984) 125

[34] P. Goddard and D. Olive, Int. Mod. Phys. Al (1986) 303;

J.K. Freericks and M.B. Halpern, LBL-25342, 1988

[35] G. Moore and N. Seiberg, Taming the conformal zoo, IASSNS-HEP-89/6;

D. Gepner, Field identification in coset conformal field theories, PUPT-1114

[36] S. Kumar, J. Diff. Geom. 20 (1984) 389

[37] R. Bott, in Representation theory of lie groups, London Math. Soc. Lect. Notes Ser. 34 (Cambridge Univ. Press, Cambridge 1979)

[38] I.N. Bernstein, I.M. Gelfand and S.I. Gelfand, in Lie groups and their representations, Summer School of the Bolyai Janos, Math. Soc., ed. by I.M. Gelfand (Wiley, New York, 1975)

[39] B. Kostant, private communication

[40] R. Bott, Bull. Soc. Math. France, 84 (1954) 251

[41] B. Kostant, Am. J. Math 81 (1959) 973

[42] N.R. Wallach, Harmonic analysis on homogeneous spaces (Marcel Dekker, New York 1973)

[43] E. Witten, Quantum field theory and the Jones polynomial, IASSNS-HEP-88/33

[44] P. Candelas, Nucl. Phys. B298 (1988) 458 